\title{
A nonsmooth model for discontinuous shear thickening fluids: Analysis and numerical solution
}

\author{
JUAN CARLOS DE LOS REYES \\ Research Center on Mathematical Modeling (MODEMAT), EPN Quito, Quito, Ecuador \\ E-mail: juan.delosreyes@epn.edu.ec \\ GEORG STADLER \\ Courant Institute of Mathematical Sciences, New York University, New York, NY, USA \\ E-mail: stadler@cims.nyu.edu
}

[Received 13 September 2013 and in revised form 22 July 2014]

\begin{abstract}
We propose a nonsmooth continuum mechanical model for discontinuous shear thickening flow. The model obeys a formulation as energy minimization problem and its solution satisfies a Stokes type system with a nonsmooth constitute relation. Solutions have a free boundary at which the behavior of the fluid changes. We present Sobolev as well as Hölder regularity results and study the limit of the model as the viscosity in the shear thickened volume tends to infinity. A mixed problem formulation is discretized using finite elements and a semismooth Newton method is proposed for the solution of the resulting discrete system. Numerical problems for steady and unsteady shear thickening flows are presented and used to study the solution algorithm, properties of the flow and the free boundary. These numerical problems are motivated by recently reported experimental studies of dispersions with high particle-to-fluid volume fractions, which often show a sudden increase of viscosity at certain strain rates.
\end{abstract}

2010 Mathematics Subject Classification: Primary 76A05, 35B65, 49M15, 76D07

Keywords: Shear thickening; non-Newtonian fluid mechanics; variational inequality; additional regularity; mixed discretization; semismooth Newton method; fictitious domain method.

\section{Introduction}

Shear thickening fluids are a model for colloidal dispersions composed of solid particles suspended in a fluid. In dispersions with a high particle-to-fluid volume fraction, jamming between the particles at high strain rates can occur. This results in an abrupt increase in viscosity that can give the fluid a solid-like behavior. To distinguish this behavior from more smooth shear thickening processes, experimental physicists have introduced the nomenclature discontinuous shear thickening.

An example for a colloidal dispersion is a cornstarch-water mixture, whose startling properties are the subject of many popular science experiments. Other examples for shear thickening fluids are cements and clays, and this non-Newtonian behavior can also be engineered into products such as pastes and paints. It has, amongst others, applications for building vibration and shock absorbing devices, and in the development of protective clothing. However, shear thickening can also be an unwanted effect that can cause severe problems in production processes [39].

The behavior of a particle-fluid mixture can be modeled as non-Newtonian fluid with a shear thickening constitutive relation. While such a rheological behavior cannot be derived from first principles, experimental studies suggest that a shear thickening constitutive relation is a reasonable 
model across a range of different particle sizes, particle shapes, and volume fractions [2, 4, 5, 12 , $29,39]$. Thus, we consider a Stokes fluid in a two- or three-dimensional domain $\Omega$, which shear thickens at high shear strain rates. We consider the equations for the conservation of momentum and mass given by

$$
\begin{aligned}
-\operatorname{Div} \sigma=f & \text { in } \Omega, \\
\nabla \cdot \boldsymbol{u}=0 & \text { in } \Omega,
\end{aligned}
$$

where $\boldsymbol{u}$ is the velocity, $\boldsymbol{\sigma}$ the stress tensor and $\boldsymbol{f}$ an external forcing. To specify the constitutive relation, we denote by $\varepsilon$ the mapping from velocity to the shear strain rate, and denote the square root of the second invariant of the strain rate tensor by $|\mathcal{E} \cdot|$, i.e.,

$$
\varepsilon \boldsymbol{u}:=\frac{1}{2}\left(\nabla \boldsymbol{u}+\nabla \boldsymbol{u}^{T}\right), \quad|\varepsilon \boldsymbol{u}|=(\mathcal{E} \boldsymbol{u}: \varepsilon \boldsymbol{\varepsilon})^{\frac{1}{2}},
$$

where ":" denotes the inner product between two second-order tensors. Of our particular interest is the sudden increase of viscosity in colloidal fluids at a certain strain rate. Thus, we consider a constitutive law that relates the shear strain rate tensor $\boldsymbol{\varepsilon} \boldsymbol{u}$ to the stress tensor $\boldsymbol{\sigma}$ as follows:

$$
\boldsymbol{\sigma}= \begin{cases}2 \mu \varepsilon \boldsymbol{u}-p \mathrm{I} & \text { if }|\varepsilon \boldsymbol{u}| \leqslant g, \\ 2\left(\mu+v-\frac{v g}{|\varepsilon \boldsymbol{u}|}\right) \varepsilon \boldsymbol{u}-p \text { I } & \text { if }|\varepsilon \boldsymbol{u}|>g .\end{cases}
$$

Here, $\mu>0, v \geqslant 0$ are viscosities, $g>0$ is the parameter at which the viscosity increases, and $p$ denotes the pressure. In the sketches in Figure 1, the constitutive relation (1d) is compared to the constitutive relation for a linear Stokes flow model. The relation (1d) corresponds to a fluid, in which a sudden viscosity increase occurs as the shear strain rate exceeds $g$. Note that the term $v g /|\boldsymbol{\varepsilon} \boldsymbol{u}|$ in (1d) is necessary to ensure continuity of the strain rate-to-stress relation, as can be seen from Figure 1.

Arguably, (1) is one of the simplest models for viscous flow with shear thickening. It involves only the parameter $g$, the viscosity $\mu$ for shear strain rates below $g$, and the viscosity increase $v$ for shear strain rates above $g$. As an alternative to (1), models with a smooth, for instance power-law shear thickening constitutive relation can be considered. Such smoother constitutive relations often involve more model parameters than our idealized model (1d). Moreover, the analysis of power-law (and other) shear thickening models requires Banach space theory (see, e.g., [13, 24, 33, 35, 36]), while (1) can be formulated and analyzed in a Hilbert space framework. Our model (1) naturally lends itself to a free boundary value problem, where the boundary separates regions with different viscosities.

Next, we summarize related work, our main contributions, and the limitations of this work.

Related Work. Understanding the phenomena responsible for shear thickening in suspensions is currently the subject of lively debates $[2,4,5,12,22,29,40]$. Possible explanations include the formation of particle hydroclusters, the expansion of particle collections when flowing past each other and compression-related jamming. The non-Newtonian behavior of shear thickening has severe implications for force transmissions and fluid-solid interactions. In [28], for instance, the authors study the focused force transmission through a cornstarch solution onto a containing wall. In [40], the fluid solidification under the impact of a solid rod is studied, and the results suggest that compression plays a role in shear thickening. The authors of [38] experimentally study 

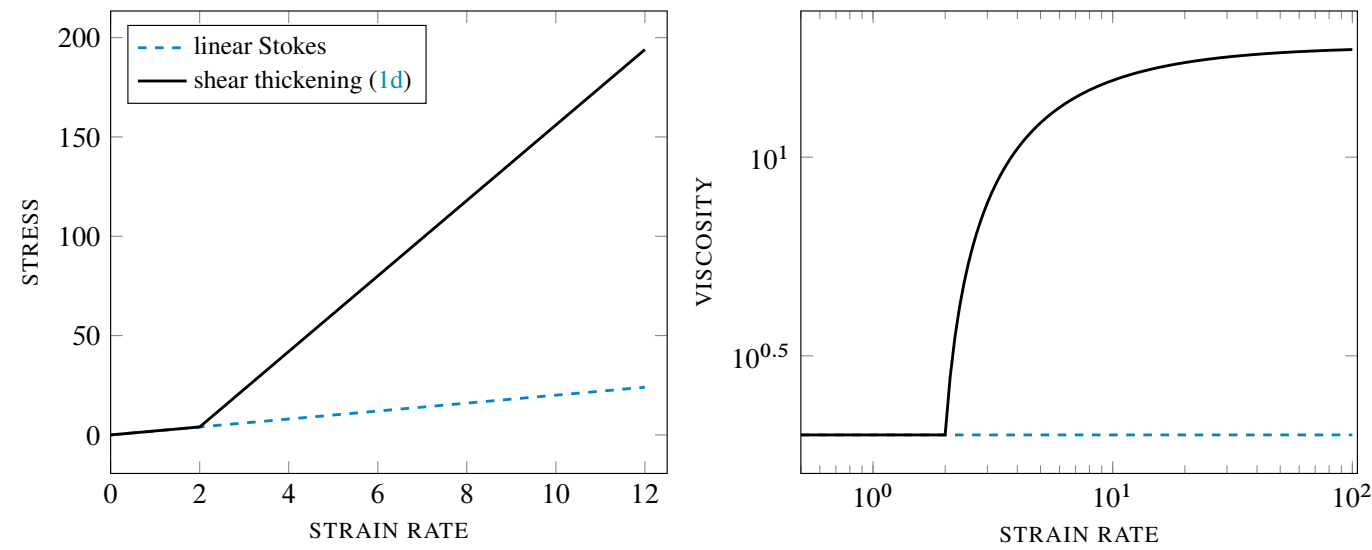

FIG. 1. Illustration of constitutive relations for Newtonian fluid with constant viscosity (dashed lines) and a shear thickening fluid (solid lines) based on (1d) with $g=2, \mu=2$ and $v=20$. Shown are the correspondence between shear strain rate and shear stress (left), and the viscosity as a function of the shear strain rate in a log-log plot (right); compare with experimental measurements, for instance [39, Figures 1 and 2], [29, Figure 2], [11, Figure 11], [12, Figure 2]. Note that the shear thickening relation (1d) contains the linear Stokes model as a special case for $v=0$ or $g \rightarrow \infty$. In Section 3, we study the behavior of the model in the perfect shear thickening case, i.e., as $v \rightarrow \infty$.

the settling of a solid sphere in a cornstarch suspension, and find a stop-and-go behavior of the sinker. A similar constitutive relation as (1d) occurs in Bingham flow. While Bingham fluids and smooth shear thinning fluids have been studied extensively in the literature [7, 17, 19], much fewer theoretical and numerical studies of continuum mechanical models for shear thickening flows are available. From a theoretical perspective, the "perfect shear thickening" limit case as $v \rightarrow \infty$ is related to the elastic-plastic torsion problem [3, 17, 23]. A related limit problems is also studied in the recent preprint [34], which appeared after the completion of this work.

Contributions. To the best of our knowledge, the proposed continuum-mechanical flow model for so-called discontinuous shear thickening has not been studied in the mathematical literature before. We derive and analyze the problem that arises from the proposed model when the viscosity for high shear rates tends to infinity, which is a variational inequality. Techniques from regularity theory are adopted to prove Sobolev as well as Hölder regularity results for the solutions. This requires the extension of regularity results for smooth potentials to potentials that contain a $C^{1,1}$-regular term. The proposed mixed discretization combined with the Newton-type solution algorithm provide an efficient method to solve the problem numerically. Using numerical experiments designed after recent experimental studies from the literature, the behavior of the free boundaries in steady and unsteady shear thickening flow are studied.

Limitations. The constitutive relation in our idealized continuum mechanical model is empirical. It may be used to model the behavior of densely packed suspensions of particles in a fluid, but it does not model the microscale particle-fluid and particle-particle interactions that ultimately cause thickening. In experiments, colloidal dispersions yield shear thinning at very low strain rates (e.g., $[5,39])$ - our model does not include this behavior. 
Paper organization. We show existence and well-posedness for a steady and an unsteady version of our shear thickening flow model in the next section. In Section 3, the model and its solution in the limit for $v \rightarrow \infty$ are investigated. Section 4 is devoted to a study of the Sobolev and Hölder regularity of the solutions to our model. This rather technical section may be skipped by readers who are mainly interested in the model formulation and its numerical behavior. Section 5 presents a mixed finite element discretization and a generalized Newton method for the resulting discrete nonlinear and nonsmooth systems. Numerical problems for steady and unsteady shear thickening flow problems are presented in Section 6. Finally, in Section 7 we draw conclusions and discuss open issues and extensions of this work.

\section{Basic solution properties}

We start by establishing basic solution properties for (1) and for a time-dependent version of (1) that models unsteady shear thickening flow. Our starting point for studying (1) is an optimization formulation using the following viscous energy functional:

$$
\min _{\boldsymbol{u} \in V} \mathcal{f}(\boldsymbol{u})=\frac{\mu}{2} \int_{\Omega} \boldsymbol{\varepsilon} \boldsymbol{u}: \varepsilon \boldsymbol{u} d x+\frac{v}{2} \int_{\Omega} \max (0,|\varepsilon \boldsymbol{u}|-g)^{2} d x-\int_{\Omega} \boldsymbol{f} \cdot \boldsymbol{u} d x,
$$

where $f \in L^{2}(\Omega)^{N}$ and $V$ is defined as the space of divergence-free velocity fields with zero Dirichlet boundary conditions on $\emptyset \neq \Gamma \subset \partial \Omega$, an open subset of the boundary of $\Omega \subset \mathbb{R}^{N}$, $N \in\{2,3\}$ :

$$
V:=\left\{\boldsymbol{u} \in H^{1}(\Omega)^{N}: \nabla \cdot \boldsymbol{u}=0 \text { in } \Omega, \boldsymbol{u}=0 \text { on } \Gamma\right\} .
$$

Note that, for simplicity of the presentation, we restrict our attention to homogeneous Dirichlet boundary conditions and target (P) with $V$ defined in (2) and $\Gamma=\partial \Omega$. More general boundary conditions can be imposed in (1), for instance combinations of Dirichlet and Neumann conditions as used for the numerical examples presented in Section 6. Computing the first variation of (P) yields that the necessary (and sufficient) condition for $\boldsymbol{u}$ to be a minimizer for (P) is that $\boldsymbol{u}$ satisfies the following variational equation:

$$
\mu \int_{\Omega} \boldsymbol{\varepsilon} \boldsymbol{u}: \mathcal{E} \boldsymbol{v} d x+v \int_{\Omega} \max (0,|\varepsilon \boldsymbol{u}|-g) \frac{\boldsymbol{\varepsilon} \boldsymbol{u}}{|\mathcal{E} \boldsymbol{u}|}: \mathcal{E} \boldsymbol{v} d x=\int_{\Omega} \boldsymbol{f} \cdot \boldsymbol{v} d x \quad \text { for all } \boldsymbol{v} \in V .
$$

Introducing a dual tensor, $\boldsymbol{\tau}$, we obtain that the solution $\boldsymbol{u}$ is also characterized by the mixed (or primal-dual) system

$$
\begin{array}{cc}
\mu \int_{\Omega} \varepsilon \boldsymbol{u}: \varepsilon \boldsymbol{v} d x+\int_{\Omega} \boldsymbol{\tau}: \varepsilon \boldsymbol{v} d x=\int_{\Omega} \boldsymbol{f} \cdot \boldsymbol{v} d x \text { for all } \boldsymbol{v} \in V \\
\boldsymbol{\tau}-v \max \left(0,1-g|\varepsilon \boldsymbol{u}|^{-1}\right) \varepsilon \boldsymbol{u}=0 & \text { a.e. in } \Omega .
\end{array}
$$

The introduction of the symmetric tensor $\boldsymbol{\tau}$ allows one to write the strong form (1) in mixed form as

$$
\begin{aligned}
-\operatorname{Div}(\mu \varepsilon \boldsymbol{u}+\boldsymbol{\tau}-p \mathrm{I})=\boldsymbol{f} & \text { in } \Omega, \\
\nabla \cdot \boldsymbol{u}=0 & \text { in } \Omega,
\end{aligned}
$$

where $\tau$ satisfies (4b). Compared to (1), this alternative form is advantageous for the discretization and numerical simulation. 
In the remainder of this paper, we denote by $(\cdot, \cdot)$ the $L^{2}(\Omega)$-inner product, and denote the corresponding norm by $\|\cdot\|$. If the functions in this inner product are vector- or tensor-valued, $(\cdot, \cdot)$ amounts to the sum of the component-wise inner products. This allows us to write (4) as

$$
\begin{aligned}
\mu(\boldsymbol{\varepsilon u}, \boldsymbol{\varepsilon} \boldsymbol{v})+(\boldsymbol{\tau}, \boldsymbol{\varepsilon v}) & =(\boldsymbol{f}, \boldsymbol{v}) & & \text { for all } \boldsymbol{v} \in V, \\
\boldsymbol{\tau}-v \max \left(0,1-g|\varepsilon \boldsymbol{u}|^{-1}\right) \varepsilon \boldsymbol{u} & =0 & & \text { a.e. in } \Omega,
\end{aligned}
$$

where $\boldsymbol{\tau}$ is a symmetric tensor with components in $L^{2}(\Omega)$. To study (6), we introduce the function

$$
m(\varepsilon):=\max \left(0,1-g|\boldsymbol{\varepsilon}|^{-1}\right) \boldsymbol{\varepsilon}
$$

for tensors $\boldsymbol{\varepsilon} \in \mathbb{R}^{N \times N}$. Note that $\max \left(0,1-g|\boldsymbol{\varepsilon}|^{-1}\right)=0$ for $|\boldsymbol{\varepsilon}| \leqslant g$ and thus we define $m(\mathbf{0})=\mathbf{0}$. We first prove Lipschitz continuity of $m$. Indeed, for $\varepsilon_{1}, \varepsilon_{2} \in \mathbb{R}^{N \times N}$ one finds

$m\left(\boldsymbol{\varepsilon}_{1}\right)-m\left(\boldsymbol{\varepsilon}_{2}\right)=\left(\max \left(0,\left|\boldsymbol{\varepsilon}_{1}\right|-g\right)-\max \left(0,\left|\boldsymbol{\varepsilon}_{2}\right|-g\right)\right) \frac{\boldsymbol{\varepsilon}_{1}}{\left|\boldsymbol{\varepsilon}_{1}\right|}+\max \left(0,\left|\boldsymbol{\varepsilon}_{2}\right|-g\right)\left(\frac{\boldsymbol{\varepsilon}_{1}}{\left|\boldsymbol{\varepsilon}_{1}\right|}-\frac{\boldsymbol{\varepsilon}_{2}}{\left|\boldsymbol{\varepsilon}_{2}\right|}\right)$.

From the Lipschitz continuity of the max-function and due to $\max \left(0,\left|\boldsymbol{\varepsilon}_{2}\right|-g\right) \leqslant\left|\boldsymbol{\varepsilon}_{2}\right|$ we obtain that

$$
\begin{aligned}
\left|m\left(\varepsilon_{1}\right)-m\left(\varepsilon_{2}\right)\right| & \leqslant|| \varepsilon_{1}|-| \varepsilon_{2}||+\left|\varepsilon_{2}\right|\left|\frac{\left|\varepsilon_{2}\right| \varepsilon_{1}-\left|\varepsilon_{1}\right| \varepsilon_{2}}{\left|\varepsilon_{1}\right|\left|\varepsilon_{2}\right|}\right| \\
& \leqslant\left|\varepsilon_{1}-\varepsilon_{2}\right|+\left|\frac{\left|\varepsilon_{2}\right| \varepsilon_{1}-\left|\varepsilon_{1}\right| \varepsilon_{1}+\left|\varepsilon_{1}\right| \varepsilon_{1}-\left|\varepsilon_{1}\right| \varepsilon_{2}}{\left|\varepsilon_{1}\right|}\right| \leqslant 3\left|\varepsilon_{1}-\varepsilon_{2}\right|,
\end{aligned}
$$

which shows the Lipschitz continuity of $m$.

Next, we discuss the dependence of the solution $\boldsymbol{u}$ of (P) on the viscosity increase $v$. To highlight the dependence of solutions on $v$, we use the notation $\boldsymbol{u}=\boldsymbol{u}_{v}$ below.

THEOREM 2.1 There exists a unique solution $\boldsymbol{u}_{v} \in V$ to (6) and the mapping $v \mapsto \boldsymbol{u}_{v} \in V$ is locally Lipschitz continuous. Moreover, for $v \geqslant \bar{v}>0$, the mapping $v \mapsto \boldsymbol{u}_{v} \in V$ is globally Lipschitz continuous.

Proof. The above pointwise estimate for $\boldsymbol{m}$ shows that $\boldsymbol{u} \rightarrow m(\varepsilon \boldsymbol{u})$ is Lipschitz continuous. Moreover, $m$ is monotone since it is the derivative of a convex and differentiable term. Consequently, the operator $T: V \mapsto V^{\prime}$ defined by

$$
T(\boldsymbol{u})=\mu(\varepsilon \boldsymbol{u}, \varepsilon \cdot)+v(m(\varepsilon \boldsymbol{u}), \varepsilon \cdot)
$$

is monotone and hemicontinuous and, therefore, pseudomonotone (see, for instance, [27, p. 171]). Thus, there exists a solution $\boldsymbol{u}_{v}$ to (6). Since $T$ is strictly monotone, uniqueness of the solution follows.

To prove the local Lipschitz dependence of $\boldsymbol{u}_{v}$ on $v$, let $\boldsymbol{u}_{1}$ and $\boldsymbol{u}_{2}$ be the unique solutions of (6) (or, equivalently (3)) for $v=v_{1}$ and $v=v_{2}$, respectively. Taking the difference between (3) for $v_{1}$ and $\nu_{2}$, and using the notation $\boldsymbol{\delta}_{u}:=\boldsymbol{u}_{1}-\boldsymbol{u}_{2}$ we obtain for all $\boldsymbol{v} \in V$ that

$$
\mu\left(\varepsilon \delta_{u}, \varepsilon \boldsymbol{v}\right)+v_{1}\left(m\left(\varepsilon \boldsymbol{u}_{1}\right), \varepsilon \boldsymbol{v}\right)-v_{2}\left(m\left(\varepsilon \boldsymbol{u}_{2}\right), \varepsilon \boldsymbol{v}\right)=0 .
$$

By adding and subtracting the term $v_{1}\left(m\left(\varepsilon \boldsymbol{u}_{2}\right), \varepsilon \boldsymbol{v}\right)$ and choosing $v=\delta_{u}$, it follows that

$$
\mu\left(\varepsilon \boldsymbol{\delta}_{\boldsymbol{u}}, \varepsilon \boldsymbol{\delta}_{\boldsymbol{u}}\right)+\left(v_{1} m\left(\varepsilon \boldsymbol{u}_{1}\right)-v_{1} m\left(\varepsilon \boldsymbol{u}_{2}\right)+v_{1} m\left(\varepsilon \boldsymbol{u}_{2}\right)-v_{2} m\left(\varepsilon \boldsymbol{u}_{2}\right), \varepsilon \boldsymbol{\delta}_{\boldsymbol{u}}\right)=0 .
$$


Thanks to the monotonicity of $\varepsilon \boldsymbol{u} \mapsto M(\varepsilon \boldsymbol{u})$, we obtain that

$$
\begin{aligned}
\mu\left\|\varepsilon \delta_{\boldsymbol{u}}\right\|_{L^{2}}^{2} & \leqslant\left|v_{1}-v_{2}\right|\left|\left(\operatorname{m}\left(\varepsilon \boldsymbol{u}_{2}\right), \boldsymbol{\varepsilon} \boldsymbol{\delta}_{\boldsymbol{u}}\right)\right| \\
& \leqslant\left|v_{1}-v_{2}\right|\left\|\max \left(0,\left|\varepsilon \boldsymbol{u}_{2}\right|-g\right)\right\|_{L^{2}}\left\|\varepsilon \boldsymbol{\delta}_{\boldsymbol{u}}\right\|_{L^{2}},
\end{aligned}
$$

which implies the desired

$$
\left\|\boldsymbol{u}_{1}-\boldsymbol{u}_{2}\right\|_{V} \leqslant \frac{C}{\mu}\left|v_{1}-v_{2}\right|\left\|\max \left(0,\left|\varepsilon \boldsymbol{u}_{2}\right|-g\right)\right\|_{L^{2}},
$$

where $C>0$ is the constant arising in Korn's inequality.

For the global Lipschitz continuity, using the form of the functional $g_{v}$, we obtain for arbitrary $v>0$ that

$$
\frac{\mu}{2}\left\|\varepsilon \boldsymbol{u}_{v}\right\|^{2} \leqslant \vartheta_{v}\left(\boldsymbol{u}_{v}\right)+\left(\boldsymbol{f}, \boldsymbol{u}_{v}\right) \leqslant \vartheta_{v}(\mathbf{0})+\|\boldsymbol{f}\|\left\|\boldsymbol{u}_{v}\right\| .
$$

Using Korn's inequality, it follows that $\left\{\boldsymbol{u}_{v}\right\}_{v>0}$ is bounded in $V$. Consequently, there exists some $c_{1}>0$, independent of $v$, such that

$$
\frac{v}{2} \int_{\Omega} \max \left(0,\left|\varepsilon \boldsymbol{u}_{v}\right|-g\right)^{2} d x \leqslant g_{v}\left(\boldsymbol{u}_{v}\right)+\left(\boldsymbol{f}, \boldsymbol{u}_{v}\right) \leqslant c_{1} \quad \text { for all } \quad v \geqslant \bar{v}>0
$$

and, thus, $\left\|\max \left(0,\left|\varepsilon \boldsymbol{u}_{v}\right|-g\right)\right\|_{L^{2}}^{2} \leqslant \frac{2 c_{1}}{\bar{v}}$. This together with (9) implies the result.

Next, we introduce a time-dependent version of the shear thickening model (P), which is given by a partial differential equation of evolution type. The solution $\boldsymbol{u}=\boldsymbol{u}(x, t)$ for $(x, t) \in \Omega \times[0, T]$ satisfies

$$
\begin{aligned}
\left(\partial_{t} \boldsymbol{u}, \boldsymbol{v}\right)+\mu(\varepsilon \boldsymbol{u}, \boldsymbol{\varepsilon} \boldsymbol{v})+(\boldsymbol{\tau}, \boldsymbol{\varepsilon v}) & =(\boldsymbol{f}, \boldsymbol{v}) & & \text { for all } \boldsymbol{v} \in V, t \in(0, T), \\
\boldsymbol{\tau}-v \max \left(0,1-g|\varepsilon \boldsymbol{u}|^{-1}\right) \varepsilon \boldsymbol{u} & =0 & & \text { a. e. in } \Omega, t \in(0, T), \\
\boldsymbol{u}(0) & =\boldsymbol{u}_{0} & & \text { in } \Omega \times\{0\} .
\end{aligned}
$$

The following existence and uniqueness result holds for this initial boundary value problem.

TheOREM 2.2 Let $\boldsymbol{f} \in L^{2}\left(0, T ; V^{\prime}\right)$ and $\boldsymbol{u}_{0} \in H:=\left\{\boldsymbol{v} \in H(\operatorname{div}, \Omega): \nabla \cdot \boldsymbol{v}=0,\left.\boldsymbol{v} \cdot \boldsymbol{n}\right|_{\Gamma}=0\right\}$. Then, there exists a unique solution $\boldsymbol{u} \in L^{2}(0, T ; V)$ for (12).

Proof. The proof follows from the properties of the nonlinear term. Thanks to the monotonicity and hemicontinuity of the operator $T(\boldsymbol{u})$ defined in (8), the result follows from [27, p. 158].

Before we study the regularity of solutions to the steady shear thickening problem (P) in Section 4, we investigate the limit of the steady shear thickening problem as $v \rightarrow \infty$ in (P) in the next section.

\section{The perfect shear thickening limit case}

By "perfect shear thickening" we refer to the model in which the viscosity becomes infinite when the flow reaches the critical shear rate $g$. The corresponding energy minimization problem is obtained 
by formally letting $v \rightarrow \infty$ in (P). This results in the following constrained optimization problem for perfect shear thickening:

$$
\min _{\substack{\boldsymbol{u} \in V \\|\varepsilon \boldsymbol{u}| \leqslant g \text { a.e. }}} \frac{\mu}{2} \int_{\Omega} \varepsilon \boldsymbol{u}: \varepsilon \boldsymbol{u} d x-(\boldsymbol{f}, \boldsymbol{u}) .
$$

In this section, we characterize solutions of $\left(\mathrm{P}_{\infty}\right)$. Since the feasible set in $\left(\mathrm{P}_{\infty}\right)$ is convex and closed and the cost functional is lower semicontinuous and strictly convex, $\left(\mathrm{P}_{\infty}\right)$ admits a unique solution $\boldsymbol{u}^{*} \in V$.

The question arises if and in what sense solutions of $(\mathrm{P})$ converge to the solution $\boldsymbol{u}^{*}$ of $\left(\mathrm{P}_{\infty}\right)$. To emphasize the dependence of the solutions of (P) on $\nu$, in this section we denote solutions of (6) by $\left(\boldsymbol{u}_{v}, \boldsymbol{\tau}_{v}\right)$, and the cost functional in (P) by $g_{v}(\cdot)$. Then we have the following lemma:

LEMma 3.1 The solutions $\boldsymbol{u}_{v}$ of (P) converge to the solution $\boldsymbol{u}^{*}$ of $\left(\mathrm{P}_{\infty}\right)$ strongly in $V$ as $v \rightarrow \infty$.

Proof. Proceeding as in the proof of Theorem 2.1 we get, from the form of the energy functional $I_{v}$ and for arbitrary $v>0$, that

$$
\frac{\mu}{2}\left\|\mathcal{E} \boldsymbol{u}_{v}\right\|^{2} \leqslant g_{v}\left(\boldsymbol{u}_{v}\right)+\left(\boldsymbol{f}, \boldsymbol{u}_{v}\right) \leqslant g_{v}(\boldsymbol{0})+\|\boldsymbol{f}\|\left\|\boldsymbol{u}_{v}\right\| .
$$

Hence, the boundedness of $\left\{\boldsymbol{u}_{v}\right\}_{v>0}$ in $V$ follows. Thus, a subsequence, which we again denote by $\boldsymbol{u}_{v}$ converges weakly in $V$ to an element $\overline{\boldsymbol{u}} \in V$. Additionally, there is some $c_{1}>0$ such that

$$
\frac{v}{2} \int_{\Omega} \max \left(0,\left|\varepsilon \boldsymbol{u}_{v}\right|-g\right)^{2} d x \leqslant c_{1} \text { for all } v>0 .
$$

This implies that

$$
\int_{\Omega} \max \left(0,\left|\varepsilon \boldsymbol{u}_{v}\right|-g\right)^{2} d x \leqslant \frac{2 c_{1}}{v} \rightarrow 0 \text { as } v \rightarrow \infty,
$$

and, using the weak lower semicontinuity of the norm,

$$
\int_{\Omega} \max (0,|\mathcal{E} \overline{\boldsymbol{u}}|-g)^{2} d x \leqslant \liminf _{\nu \rightarrow \infty} \int_{\Omega} \max \left(0,\left|\mathcal{E} \boldsymbol{u}_{\nu}\right|-g\right)^{2} d x \leqslant 0,
$$

which implies that $|\varepsilon \overline{\boldsymbol{u}}| \leqslant g$ almost everywhere, yielding that $\overline{\boldsymbol{u}}$ is feasible for $\left(\mathrm{P}_{\infty}\right)$.

Next we show that $\left\|\boldsymbol{u}_{v}\right\|_{V} \rightarrow\|\overline{\boldsymbol{u}}\|_{V}$, which, together with the weak convergence implies the strong convergence of $\boldsymbol{u}_{v}$ to $\overline{\boldsymbol{u}}$ in $V$. This norm convergence follows from

$$
\begin{aligned}
\frac{\mu}{2}\|\varepsilon \overline{\boldsymbol{u}}\|^{2} & \leqslant \liminf _{\nu \rightarrow \infty} \frac{\mu}{2}\left\|\varepsilon \boldsymbol{u}_{v}\right\|^{2} \leqslant \liminf _{\nu \rightarrow \infty}\left\{g_{\nu}\left(\boldsymbol{u}_{v}\right)+\left(\boldsymbol{f}, \boldsymbol{u}_{v}\right)\right\} \\
& \leqslant \liminf _{\nu \rightarrow \infty}\left\{\mathcal{G}_{\nu}(\overline{\boldsymbol{u}})+\left(\boldsymbol{f}, \boldsymbol{u}_{v}\right)\right\}=g(\overline{\boldsymbol{u}})+(\boldsymbol{f}, \overline{\boldsymbol{u}})=\frac{\mu}{2}\|\mathcal{E} \overline{\boldsymbol{u}}\|^{2},
\end{aligned}
$$

where we used the weakly lower semicontinuity of the norm as well as $|\mathcal{E} \overline{\boldsymbol{u}}| \leqslant g$ almost everywhere. Thus, $\boldsymbol{u}_{v} \rightarrow \overline{\boldsymbol{u}}$ strongly in $V$. It remains to show that $\overline{\boldsymbol{u}}$ is the solution to $\left(\mathrm{P}_{\infty}\right)$. As shown above, $|\mathcal{E} \overline{\boldsymbol{u}}| \leqslant g$ almost everywhere in $\Omega$, and thus $\overline{\boldsymbol{u}}$ is feasible for $\left(\mathrm{P}_{\infty}\right)$. Using the solution $\boldsymbol{u}^{*}$ of $\left(\mathrm{P}_{\infty}\right)$, we obtain

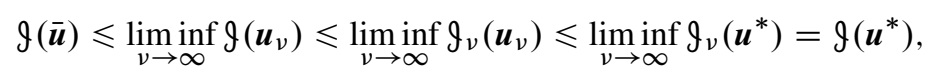

and thus $\overline{\boldsymbol{u}}=\boldsymbol{u}^{*}$. This shows that $\boldsymbol{u}^{*}$ is unique and every subsequence of $\left\{\boldsymbol{u}_{v}\right\}_{v>0}$ converges to $\boldsymbol{u}^{*}$, which completes the proof. 
Next, we derive an optimality system that characterizes the solution to $\left(\mathrm{P}_{\infty}\right)$. To do that, we consider the following space:

$$
\varepsilon V:=\left\{\boldsymbol{w} \in L^{2}\left(\Omega, \mathbb{R}^{N \times N}\right): \boldsymbol{w}=\varepsilon \boldsymbol{v} \text { for some } \boldsymbol{v} \in V\right\},
$$

endowed with the $L^{2}-$ Frobenius norm

$$
\|\boldsymbol{w}\|_{\varepsilon V}=\left(\int_{\Omega} \operatorname{tr}\left(\boldsymbol{w}^{T} \boldsymbol{w}\right) d x\right)^{1 / 2} .
$$

The dual space is denoted by $(\varepsilon V)^{\prime}$. Since $\varepsilon V$ is a closed subspace of $L^{2}\left(\Omega, \mathbb{R}^{N \times N}\right)$, it constitutes itself a Hilbert space.

THEOREM 3.2 A subsequence of $\left\{\boldsymbol{\tau}_{v}\right\}_{v>0}$ converges weakly in $(\varepsilon V)^{\prime}$ to a tensor $\boldsymbol{\tau}^{*}$, and $\left(\boldsymbol{u}^{*}, \boldsymbol{\tau}^{*}\right)$ satisfies the following system:

$$
\begin{aligned}
\mu\left(\varepsilon \boldsymbol{u}^{*}, \boldsymbol{\varepsilon} \boldsymbol{v}\right)+\left\langle\boldsymbol{\tau}^{*}, \boldsymbol{\varepsilon} \boldsymbol{v}\right\rangle_{(\varepsilon V)^{\prime}} & =(\boldsymbol{f}, \boldsymbol{v}) \text { for all } \boldsymbol{v} \in V \\
\left|\mathcal{E} \boldsymbol{u}^{*}\right| & \leqslant g \text { a.e. in } \Omega, \\
\left\langle\boldsymbol{\tau}^{*}, \boldsymbol{\varepsilon}\left(\boldsymbol{v}-\boldsymbol{u}^{*}\right)\right\rangle_{(\varepsilon V)^{\prime}} & \leqslant 0 \text { for all } \boldsymbol{v} \in V \text { with }|\varepsilon \boldsymbol{v}| \leqslant g \text { a.e. in } \Omega, \\
\left\langle\boldsymbol{\tau}^{*}, \boldsymbol{\varepsilon} \boldsymbol{u}^{*}\right\rangle_{(\varepsilon V)^{\prime}} & \geqslant 0 .
\end{aligned}
$$

Proof. From the boundedness of $\left\{\boldsymbol{u}_{v}\right\}_{v>0}$ (see proof of Lemma 3.1), it follows from (6a) that $\left\{\boldsymbol{\tau}_{v}\right\}_{v>0}$ is bounded in $(\varepsilon V)^{\prime}$. Therefore, there exists a subsequence $\left\{\boldsymbol{\tau}_{v}\right\}$ such that

$$
\boldsymbol{\tau}_{v} \rightarrow \boldsymbol{\tau}^{*} \text { weakly in }(\varepsilon V)^{\prime} .
$$

Passing to the limit in (6a), we obtain

$$
\mu\left(\varepsilon \boldsymbol{u}^{*}, \boldsymbol{\varepsilon} \boldsymbol{v}\right)+\left\langle\boldsymbol{\tau}^{*}, \boldsymbol{\varepsilon} \boldsymbol{v}\right\rangle_{(\varepsilon V)^{\prime}}=(\boldsymbol{f}, \boldsymbol{v}) \text { for all } \boldsymbol{v} \in V .
$$

In Lemma 3.1 we have already shown that $\boldsymbol{u}^{*}$ is feasible for $\left(\mathrm{P}_{\infty}\right)$, which proves $(15 \mathrm{~b})$. To prove (15c), note that the optimality of $\boldsymbol{u}^{*}$ for $\left(\mathrm{P}_{\infty}\right)$ implies

$$
\mu\left(\varepsilon \boldsymbol{u}^{*}, \mathcal{E}\left(\boldsymbol{v}-\boldsymbol{u}^{*}\right)\right) \geqslant\left(\boldsymbol{f}, \boldsymbol{v}-\boldsymbol{u}^{*}\right) \text { for all } \boldsymbol{v} \in V \text { with }|\varepsilon \boldsymbol{v}| \leqslant g \text { a.e. in } \Omega .
$$

Choosing $\boldsymbol{v}:=\boldsymbol{v}-\boldsymbol{u}^{*}$ in (16), and subtracting (17) from this equation yields

$$
\left\langle\boldsymbol{\tau}^{*}, \mathcal{E}\left(\boldsymbol{v}-\boldsymbol{u}^{*}\right)\right\rangle_{(\varepsilon V)^{\prime}} \leqslant 0 \text { for all } \boldsymbol{v} \in V \text { with }|\varepsilon \boldsymbol{v}| \leqslant g \text { a.e. in } \Omega,
$$

and thus (15c). In particular, taking $\boldsymbol{v}=0$, the inequality (15d) follows.

Note that the optimality system (15) for the limit problem $\left(\mathrm{P}_{\infty}\right)$ does, in general, not allow a pointwise characterization of $\boldsymbol{\tau}^{*}$. This is in contrast to the nonlinear equation system (4), which characterizes the solution to $(\mathrm{P})$. In the next section, we study the regularity of solutions to the steady shear thickening problem $(\mathrm{P})$.

\section{Solution regularity}

Here, we investigate additional regularity for the solution of the stationary model (3). We first give a local Sobolev regularity result, and then, in Section 4.2, prove a partial Hölder regularity result. 


\subsection{Sobolev regularity}

We start by extending a result for smooth dissipative potentials, due to Fuchs and Seregin [15, Section 3], to the case of potentials constituted by the sum of a smooth coercive term and a convex $C^{1,1}$-regular term.

THEOREM 4.1 Let $\boldsymbol{u} \in V$ be a solution of the variational equation

$$
\mu(\varepsilon \boldsymbol{u}, \boldsymbol{\varepsilon} \boldsymbol{v})+\int_{\Omega} g(\varepsilon \boldsymbol{u}): \varepsilon \boldsymbol{v} d x=(\boldsymbol{f}, \boldsymbol{v}) \text { for all } \boldsymbol{v} \in V
$$

where $\mathcal{G}$ is a monotone and Lipschitz continuous operator. If $f \in L^{2}(\Omega)^{N}$, then the solution has the additional regularity $\boldsymbol{u} \in H_{l o c}^{2}(\Omega)^{N}$.

Proof. Since we target local regularity, we can assume w.l.o.g. $C^{2}$-regularity of the boundary. If $\partial \Omega$ is not that smooth, we can consider a regular subdomain $\hat{\Omega}$ of $\Omega$, extend test functions from $\hat{\Omega}$ to $\Omega$ by zero and combine the weak forms on $\hat{\Omega}$. We first consider the weak formulation of the linear Stokes equation: Find $w \in V$ such that

$$
\mu(\varepsilon \boldsymbol{w}, \varepsilon \boldsymbol{v})=(\boldsymbol{f}, \boldsymbol{v}) \text { for all } \boldsymbol{v} \in V .
$$

Due to the regularity of $\partial \Omega$, the solution $w$ of (20) has the additional regularity $w \in H^{2}(\Omega)^{N}$, and the estimate [26, Ch. 3, Sec. 5]

$$
\int_{\Omega}\left(|\nabla \boldsymbol{w}|^{2}+\left|\nabla^{2} \boldsymbol{w}\right|^{2}\right) d x \leqslant c_{1}\|f\|_{L^{2}(\Omega)}^{2}
$$

holds for a constant $c_{1}>0$ that only depends on the domain $\Omega$. The problem (19) can thus be written as

$$
\mu(\varepsilon \boldsymbol{u}, \varepsilon \boldsymbol{v})+(\mathcal{G}(\varepsilon \boldsymbol{u}), \varepsilon \boldsymbol{v})-\mu(\varepsilon \boldsymbol{w}, \varepsilon \boldsymbol{v})=0 \text { for all } \boldsymbol{v} \in V,
$$

or, equivalently, using a pressure function $p \in L^{2}(\Omega)$ (see [26, Ch. 1, Sec. 2]) as: Find $\boldsymbol{u} \in V$ such that

$$
\mu(\mathcal{E} \boldsymbol{u}, \boldsymbol{\varepsilon} \boldsymbol{v})+(\mathcal{G}(\mathcal{\varepsilon} \boldsymbol{u}), \boldsymbol{\varepsilon} \boldsymbol{v})=\mu(\mathcal{E} \boldsymbol{w}, \boldsymbol{\varepsilon} \boldsymbol{v})+(p, \nabla \cdot \boldsymbol{v})
$$

for all $\boldsymbol{v} \in H_{0}^{1}(\Omega)^{N}$. Introducing, for $h \in \mathbb{R}^{N}$ such that $x+h \in \Omega$, the difference operator $D_{h} g(x):=g(x+h)-g(x)$ and the smooth cutoff function $\varphi \in C_{0}^{2}(\Omega)$, we obtain, choosing $\boldsymbol{v}=D_{h}^{-1}\left(\varphi^{2} D_{h} \boldsymbol{u}\right)$ in $(22)$, that

$$
\begin{aligned}
\mu\left(\mathcal{E}\left(D_{h} \boldsymbol{u}\right), \mathcal{E}\left(\varphi^{2} D_{h} \boldsymbol{u}\right)\right)+([\mathcal{G}(\mathcal{E} \boldsymbol{u}(x+h)) & \left.-\mathcal{G}(\varepsilon \boldsymbol{u}(x))], \mathcal{E}\left(\varphi^{2} D_{h} \boldsymbol{u}\right)\right) \\
& =\mu\left(\varepsilon\left(D_{h} \boldsymbol{w}\right), \mathcal{E}\left(\varphi^{2} D_{h} \boldsymbol{u}\right)\right)+\left(D_{h} p, \nabla \cdot\left(\varphi^{2} D_{h} \boldsymbol{u}\right)\right) .
\end{aligned}
$$

Moving $\varphi$ outside of $\varepsilon$, this yields

$$
\begin{array}{r}
\mu\left(\varphi^{2} \varepsilon\left(D_{h} \boldsymbol{u}\right), \varepsilon\left(D_{h} \boldsymbol{u}\right)\right)+\left(\varphi^{2}[\mathcal{G}(\mathcal{E} \boldsymbol{u}(x+h))-\mathcal{G}(\varepsilon \boldsymbol{u}(x))], \mathcal{E}\left(D_{h} \boldsymbol{u}\right)\right) \\
=-2 \mu\left(\varphi \varepsilon\left(D_{h} \boldsymbol{u}\right),\left(D_{h} \boldsymbol{u} \odot \nabla \varphi\right)\right)-2\left(\varphi[\mathcal{G}(\mathcal{E} \boldsymbol{u}(x+h))-\mathcal{G}(\varepsilon \boldsymbol{u}(x))],\left(D_{h} \boldsymbol{u} \odot \nabla \varphi\right)\right) \\
\mu\left(\varphi^{2} \varepsilon\left(D_{h} \boldsymbol{w}\right), \mathcal{E}\left(D_{h} \boldsymbol{u}\right)\right)+2 \mu\left(\varphi \varepsilon\left(D_{h} \boldsymbol{w}\right),\left(D_{h} \boldsymbol{u} \odot \nabla \varphi\right)\right)+\left(D_{h} p, \nabla \cdot\left(\varphi^{2} D_{h} \boldsymbol{u}\right)\right),
\end{array}
$$


where $(\boldsymbol{a} \odot \boldsymbol{b})_{i j}=\frac{1}{2}\left(a_{i} b_{j}+a_{j} b_{i}\right)$ for $\boldsymbol{a}, \boldsymbol{b} \in \mathbb{R}^{N}$. Using the monotonicity of $\mathcal{G}$ on the left hand side and its Lipschitz continuity on the right hand side, we get the estimate:

$$
\begin{gathered}
\left\|\varphi \varepsilon\left(D_{h} \boldsymbol{u}\right)\right\|_{L^{2}}^{2} \leqslant c_{2}\left\{\left\|\varphi \varepsilon\left(D_{h} \boldsymbol{u}\right)\right\|_{L^{2}}\left(\int_{\Omega}\left|D_{h} \boldsymbol{u}\right|^{2}|\nabla \varphi|^{2} d x\right)^{1 / 2}\right. \\
+\left\|\varphi \varepsilon\left(D_{h} \boldsymbol{u}\right)\right\|_{L^{2}}\left\|\varphi \varepsilon\left(D_{h} \boldsymbol{w}\right)\right\|_{L^{2}} \\
\left.\quad+\int_{\Omega} \varphi\left|\varepsilon\left(D_{h} \boldsymbol{w}\right)\left\|D_{h} \boldsymbol{u}\right\| \nabla \varphi\right| d x+\left(D_{h} p, \nabla \cdot\left(\varphi^{2} D_{h} \boldsymbol{u}\right)\right)\right\}
\end{gathered}
$$

for some constant $c_{2}>0$. Following [15, Thm. 3.1], for a constant $c_{3}>0$ the estimate

$$
\left(D_{h} p, \nabla \cdot\left(\varphi^{2} D_{h} \boldsymbol{u}\right)\right) \leqslant c_{3}|h|\|p\|_{L^{2}}\left\|\varepsilon\left(\varphi D_{h} \boldsymbol{u}\right)\right\|_{L^{2}}
$$

holds. Together with (23), this implies

$$
\left\|\varphi \varepsilon\left(D_{h} \boldsymbol{u}\right)\right\|_{L^{2}}^{2} \leqslant c_{4} \int_{\Omega}\left|\varepsilon\left(D_{h} \boldsymbol{w}\right)\right|^{2}+\left|D_{h} \boldsymbol{u}\right|^{2}+|h|^{2}|p|^{2} d x
$$

for a constant $c_{4}>0$. Consequently, from standard pressure estimates and the properties of the difference operator (see [10, pg. 277]) we obtain that

$$
\int_{\Omega} \varphi^{2}|\nabla \varepsilon(\boldsymbol{u})|^{2} d x \leqslant c_{5} \int_{\Omega}|\boldsymbol{f}|^{2} d x .
$$

with $c_{5}>0$, which implies the result.

Taking $G(\varepsilon \boldsymbol{u}):=m(\varepsilon \boldsymbol{u})=v \max \left(0,1-g|\varepsilon \boldsymbol{u}|^{-1}\right) \mathcal{E} \boldsymbol{u}$ we obtain the desired regularity for solutions to (P) (or, equivalently, (3)) as direct consequence of Theorem 4.1.

COROLlary 4.2 Let $\boldsymbol{f} \in L^{2}(\Omega)^{N}$. Then the unique solution $\boldsymbol{u} \in V$ to (3) has the additional regularity $\boldsymbol{u} \in H_{l o c}^{2}(\Omega)^{N}$.

\subsection{Hölder regularity}

Next we prove a partial Hölder regularity result for the solution of $(\mathrm{P})$. For that purpose, we consider an auxiliary regularized version of $(\mathrm{P})$, for which regularity is obtained from results for minimizers of variational problems with $C^{2}$-potentials. Then, an asymptotic analysis is performed in order to prove the claim. The regularized minimization problem is

$$
\min _{\boldsymbol{u} \in V} g_{\delta}(\boldsymbol{u}):=\frac{\mu}{2} \int_{\Omega} \varepsilon \boldsymbol{u}: \varepsilon \boldsymbol{u} d x+\frac{\nu}{2} \int_{\Omega} \max _{\delta}(0,|\varepsilon \boldsymbol{u}|-g)^{2} d x-\int_{\Omega} \boldsymbol{f} \cdot \boldsymbol{u} d x,
$$

where

$$
\max _{\delta}(0, x)=\left\{\begin{array}{lll}
x & \text { if } \quad x \geqslant \delta \\
-\frac{1}{16 \delta^{3}} x^{4}+\frac{3}{8 \delta^{2}} x^{2}+\frac{1}{2} x+\frac{3 \delta}{16} & \text { if } \quad|x| \leqslant \delta \\
0 & \text { if } \quad x \leqslant-\delta
\end{array}\right.
$$


is a twice continuously differentiable approximation of the max-function. The necessary and sufficient optimality condition for the minimizer $\boldsymbol{u}_{\delta}$ of $\left(P_{\delta}\right)$ is given by the following variational equation:

$$
\begin{aligned}
\mu \int_{\Omega} \boldsymbol{\varepsilon} \boldsymbol{u}_{\delta}: \varepsilon \boldsymbol{v} d x+v \int_{\Omega} \max _{\delta}\left(0,\left|\boldsymbol{\varepsilon} \boldsymbol{u}_{\delta}\right|-g\right) \mathbf{1}_{\delta}\left(\left|\boldsymbol{\varepsilon} \boldsymbol{u}_{\delta}\right|\right. & -g) \frac{\boldsymbol{\varepsilon} \boldsymbol{u}_{\delta}}{\left|\boldsymbol{\varepsilon} \boldsymbol{u}_{\delta}\right|}: \boldsymbol{\varepsilon} \boldsymbol{v} d x \\
& =\int_{\Omega} \boldsymbol{f} \cdot \boldsymbol{v} d x \text { for all } \boldsymbol{v} \in V,
\end{aligned}
$$

where $\mathbf{1}_{\delta}(x)$ is the derivative of $\max _{\delta}(0, x)$. To apply the regularity result in [14, Thm. 2.1] to $\left(P_{\delta}\right)$, the following growth condition for the second derivative of

$$
m_{\delta}(\boldsymbol{\varepsilon}):=\frac{1}{2} \max _{\delta}(0,|\boldsymbol{\varepsilon}|-g)^{2} \text { for } \boldsymbol{\varepsilon} \in \mathbb{R}^{N \times N}
$$

is used; for the simple proof we refer to Appendix A.

Lemma 4.3 There exists a constant $C>0$ independent of $\delta$ and $\varepsilon$ such that

$$
\left|\frac{\partial^{2} m_{\delta}(\boldsymbol{\varepsilon})}{\partial \boldsymbol{\varepsilon}^{2}}\right| \leqslant C
$$

Therefore (see [16, Thm. 3.1.5]), we obtain that the solution $\boldsymbol{u}_{\delta}$ of $\left(P_{\delta}\right)$ is in $C^{1, \alpha}\left(\Omega^{*}\right), 0<$ $\alpha<1$, where

$$
\Omega^{*}:=\left\{x \in \Omega: x \text { is a Lebesgue point of } \mathcal{E} \boldsymbol{u} \text { and } \lim _{r \rightarrow+0} V\left(\boldsymbol{u}, B_{r}(x)\right)=0\right\},
$$

and $\Omega^{*}$ is open. Above, $B_{r}(x)$ is the ball with radius $r$ centered at $x$, and

$$
\mathcal{V}\left(\boldsymbol{u}, B_{r}(x)\right):=f_{B_{r}(x)}\left|\mathcal{E} \boldsymbol{u}-(\varepsilon \boldsymbol{u})_{x, r}\right|^{2} d z \text { and }(\varepsilon \boldsymbol{u})_{x, r}:=f_{B_{r}(x)} \varepsilon \boldsymbol{u} d z .
$$

If $n=2$, then $\boldsymbol{u}_{\delta}$ belongs to the space $C^{1, \alpha}(\Omega), 0<\alpha<1$ (see [14, Thm. 4.1]).

Next, we study the regularity in the limit as $\delta \rightarrow 0$. In the following lemma, we establish a pointwise convergence result for the regularization of the max-function; for the proof we refer to Appendix A.

Lemma 4.4 For $\delta \rightarrow \infty$,

$$
\left|\max (0, x)-\max _{\delta}(0, x) \mathbf{1}_{\delta}(x)\right| \rightarrow 0 \text { uniformly in } x \in \mathbb{R} .
$$

In order to prove partial regularity, the key ingredient is a blow-up estimate, which is verified in the next lemma. Along the proof, which can be found in Appendix A, we make use of results which can be traced back to [14]. In fact, we only give a detailed justification of the main steps, where the proof differs from the one in [14].

Lemma 4.5 (Blow-up) Let $\sigma>0$ and let $\boldsymbol{u}_{\delta}$ be the minimizer for $g_{\delta}$ in $V$. Then there exists a constant $c_{0}$ such that for every $t \in(0,1)$ there exists $\epsilon$, depending on $t$ and $\sigma$, such that

$$
\left|\left(\varepsilon \boldsymbol{u}_{\delta}\right)_{x_{0}, r}\right| \leqslant \sigma \text { and } v\left(\boldsymbol{u}_{\delta}, B_{r}\left(x_{0}\right)\right)<\epsilon^{2},
$$

for some ball $B_{r}\left(x_{0}\right) \subset \Omega$, imply the estimate

$$
v\left(\boldsymbol{u}_{\delta}, B_{t r}\left(x_{0}\right)\right) \leqslant c_{0} t^{2} v\left(\boldsymbol{u}_{\delta}, B_{r}\left(x_{0}\right)\right) .
$$


The blow-up lemma is the main tool to prove the Hölder regularity stated in the next theorem.

THEOREM 4.6 Let $\boldsymbol{u} \in V$ be the minimizer of (P). Then $\Omega^{*}$ is open and $\varepsilon \boldsymbol{u} \in C^{0, \alpha}\left(\Omega^{*}\right)$ for every $0<\alpha<1$.

Proof. Step 1. We start by proving strong convergence of the minimizers of $\left(P_{\delta}\right)$ towards the minimizer of (P) in $V$ as $\delta \rightarrow 0$. Taking the difference between the variational equations (3) and (24) and testing with $\boldsymbol{v}=\boldsymbol{u}-\boldsymbol{u}_{\delta}$ we obtain that

$$
\begin{aligned}
\mu \int_{\Omega}\left|\varepsilon \boldsymbol{u}-\varepsilon \boldsymbol{u}_{\delta}\right|^{2} d x & +v \int_{\Omega}\left[\max (0,|\varepsilon \boldsymbol{u}|-g) \frac{\varepsilon \boldsymbol{u}}{|\varepsilon \boldsymbol{u}|}\right. \\
& \left.-\max _{\delta}\left(0,\left|\varepsilon \boldsymbol{u}_{\delta}\right|-g\right) \mathbf{1}_{\delta}\left(\left|\varepsilon \boldsymbol{u}_{\delta}\right|-g\right) \frac{\varepsilon \boldsymbol{u}_{\delta}}{\left|\varepsilon \boldsymbol{u}_{\delta}\right|}\right]:\left(\varepsilon \boldsymbol{u}-\varepsilon \boldsymbol{u}_{\delta}\right) d x=0 .
\end{aligned}
$$

Introducing for $v \in V$ the notation $\widetilde{\max }(\boldsymbol{v}):=\max (0,|\varepsilon \boldsymbol{v}|-g)$ and $\widetilde{\max _{\delta}}(\boldsymbol{v}):=\max _{\delta}(0,|\varepsilon \boldsymbol{v}|-g)$, we get that

$$
\begin{aligned}
\mu \int_{\Omega}\left|\varepsilon \boldsymbol{u}-\varepsilon \boldsymbol{u}_{\delta}\right|^{2} d x+v \int_{\Omega}\left[\widetilde{\max }(\boldsymbol{u}) \frac{\varepsilon \boldsymbol{u}}{|\varepsilon \boldsymbol{u}|}-\widetilde{\max }(\boldsymbol{u}) \frac{\varepsilon \boldsymbol{u}_{\delta}}{\left|\varepsilon \boldsymbol{u}_{\delta}\right|}\right. \\
\left.+\widetilde{\max }(\boldsymbol{u}) \frac{\varepsilon \boldsymbol{u}_{\delta}}{\left|\varepsilon \boldsymbol{u}_{\delta}\right|}-\widetilde{\max _{\delta}}\left(\boldsymbol{u}_{\delta}\right) \mathbf{1}_{\delta}\left(\left|\varepsilon \boldsymbol{u}_{\delta}\right|-g\right) \frac{\varepsilon \boldsymbol{u}_{\delta}}{\left|\varepsilon \boldsymbol{u}_{\delta}\right|}\right]:\left(\varepsilon \boldsymbol{u}-\varepsilon \boldsymbol{u}_{\delta}\right) d x=0
\end{aligned}
$$

which, thanks to the monotonicity of the operator $m$, implies that

$$
\mu \int_{\Omega}\left|\boldsymbol{\varepsilon} \boldsymbol{u}-\varepsilon \boldsymbol{u}_{\delta}\right|^{2} d x \leqslant v \int_{\Omega}\left|\widetilde{\max }(\boldsymbol{u})-\widetilde{\max }\left(\boldsymbol{u}_{\delta}\right) \mathbf{1}_{\delta}\left(\left|\varepsilon \boldsymbol{u}_{\delta}\right|-g\right)\right|\left|\varepsilon \boldsymbol{u}-\varepsilon \boldsymbol{u}_{\delta}\right| d x .
$$

From the approximation properties of the $\max _{\delta}$ function (see Lemma 4.4) we finally get that

$$
\left\|\widetilde{\max }(\boldsymbol{u})-\widetilde{\max _{\delta}}\left(\boldsymbol{u}_{\delta}\right) \mathbf{1}_{\delta}\left(\left|\varepsilon \boldsymbol{u}_{\delta}\right|-g\right)\right\|_{L^{2}} \rightarrow 0 \text { as } \delta \rightarrow 0
$$

and, by Korn's inequality, $\boldsymbol{u}_{\delta} \rightarrow \boldsymbol{u}$ in $V$ as $\delta \rightarrow 0$.

Step 2. Let $x_{0} \in \Omega^{*}$. Choosing a radius $r$ such that $\left|(\mathcal{E} \boldsymbol{u})_{x_{0}, r}\right|<\sigma / 2$ and $\mathcal{V}\left(\boldsymbol{u}, B_{r}\left(x_{0}\right)\right)<\tilde{\epsilon}(t)$ with $\tilde{\epsilon}(t)=\epsilon^{2}(t)$, as well as $t$ such that $c_{0} t^{2} \leqslant \frac{1}{2}$, it follows, for $\delta$ small enough, that

$$
\left|\left(\varepsilon \boldsymbol{u}_{\delta}\right)_{x_{0}, r}\right|<\frac{\sigma}{2}, \quad v\left(\boldsymbol{u}_{\delta}, B_{r}\left(x_{0}\right)\right)<\tilde{\epsilon}(t)
$$

and, by [14, Lem. 2.5],

$$
v\left(\boldsymbol{u}_{\delta}, B_{t r}\left(x_{0}\right)\right) \leqslant \frac{1}{2} v\left(\boldsymbol{u}_{\delta}, B_{r}\left(x_{0}\right)\right) .
$$

Thanks to Lemma 4.5, a blow up estimate holds for the whole sequence $\left\{\boldsymbol{u}_{\delta}\right\}$ with constants independent of $\delta$. Arguing as in [14, p. 1011] we thus obtain that

$$
\vartheta\left(\boldsymbol{u}_{\delta}, B_{t^{k}}\left(x_{0}\right)\right) \leqslant 2^{-k} \vartheta\left(\boldsymbol{u}_{\delta}, B_{r}\left(x_{0}\right)\right),
$$

for all $k$ and $\delta$ small enough. Hence, $\varepsilon \boldsymbol{u}_{\delta} \in C^{0, \alpha}\left(B_{r / 2}\left(x_{0}\right)\right), 0<\alpha<1$ and, by passing to the limit, it follows that $\varepsilon \boldsymbol{u} \in C^{0, \alpha}\left(B_{r / 2}\left(x_{0}\right)\right), 0<\alpha<1$.

After these regularity results, in the next section we discuss the numerical discretization of the shear thickening flow problem (P) and present an efficient solution method to solve the resulting nonlinear (and nonsmooth) finite-dimensional problem. 


\section{Discretization and Newton-type solution algorithm}

Next, in Section 5.1, we present a mixed finite element discretization for our model for steady and unsteady discontinuous thickening flows. For the application of the finite element method to free boundary problems we refer for instance to $[8,25]$. In Section 5.2, we propose a generalized Newton algorithm that enables an efficient solution of the arising discrete nonlinear systems.

\subsection{Finite element discretization}

We use finite elements to discretize the mixed system (6). For that purpose, we introduce the finite element spaces $W_{h} \subset H^{1}(\Omega)^{N}$ for the velocity, $P_{h} \subset L^{2}(\Omega)$ for the pressure and $Q_{h} \subset L^{2}(\Omega)^{s}$ for the dual tensor, and we assume that $W_{h}$ and $P_{h}$ satisfy the inf-sup condition for stability of numerical methods for saddle point problems [9]. Using the symmetry of the dual tensor, it is sufficient to approximate $s=3$ (rather than all four) components of $\boldsymbol{\tau}$ in two dimensions, and $s=6$ (rather than nine) stress components in three dimension. We first target the discretization of the steady shear thickening flow problem and then consider the time-dependent problem.

The finite element approximation of (6) is given by: Find $\left(\boldsymbol{u}_{h}, p_{h}, \boldsymbol{\tau}_{h}\right) \in W_{h} \times P_{h} \times Q_{h}$ such that for all $\left(\boldsymbol{v}_{h}, q_{h}, \varphi_{h}\right) \in W_{h} \times P_{h} \times Q_{h}$ :

$$
\begin{array}{r}
\mu\left(\varepsilon \boldsymbol{u}_{h}, \boldsymbol{\varepsilon} \boldsymbol{v}_{h}\right)+\left(\boldsymbol{\tau}_{h}, \boldsymbol{\varepsilon} \boldsymbol{v}_{h}\right)+\left(p_{h}, \nabla \cdot \boldsymbol{v}_{h}\right)+\left(q_{h}, \nabla \cdot \boldsymbol{u}_{h}\right)-\left(\boldsymbol{f}, \boldsymbol{v}_{h}\right)=0 \\
v\left(\max \left(0,1-g\left|\varepsilon \boldsymbol{u}_{h}\right|^{-1}\right) \boldsymbol{\varepsilon} \boldsymbol{u}_{h}, \boldsymbol{\varphi}_{h}\right)-\left(\boldsymbol{\tau}_{h}, \boldsymbol{\varphi}_{h}\right)=0 .
\end{array}
$$

We use numerical quadrature to approximate the integrals in (29) and denote by $(\underline{\boldsymbol{u}}, \underline{\boldsymbol{p}}, \underline{\boldsymbol{\tau}}) \in \mathbb{R}^{m} \times$ $\mathbb{R}^{n} \times \mathbb{R}^{l}$ the finite element coefficient vectors for $\left(\boldsymbol{u}_{h}, p_{h}, \boldsymbol{\tau}_{h}\right)$. This results in the following nonlinear system:

$$
\left[\begin{array}{ccc}
\mathbf{A} & \mathbf{B}^{T} & \mathbf{G}^{T} \\
\mathbf{B} & \mathbf{0} & \mathbf{0} \\
\tilde{\mathbf{G}}(\underline{\boldsymbol{u}}) & \mathbf{0} & -\mathbf{N}
\end{array}\right]\left[\begin{array}{c}
\underline{\boldsymbol{u}} \\
\underline{p} \\
\underline{\boldsymbol{\tau}}
\end{array}\right]=\left[\begin{array}{c}
\frac{f}{\mathbf{0}} \\
\mathbf{0}
\end{array}\right] .
$$

Here, $\mathbf{A} \in \mathbb{R}^{m \times m}$ and $\mathbf{B}^{n \times m}$ correspond to the discretization of the viscous and the divergence operators, respectively. Moreover, $\mathbf{N} \in \mathbb{R}^{l \times l}$ is a mass matrix in $Q_{h}$, and

$$
\mathbf{G}=\mathbf{F}^{T} \operatorname{diag}(\boldsymbol{\omega}) \mathbf{E} \text { and } \tilde{\mathbf{G}}(\underline{\boldsymbol{u}})=\mathbf{F}^{T} \operatorname{diag}(\boldsymbol{\omega}) \operatorname{diag}(\varrho(\underline{\boldsymbol{u}})) \mathbf{E},
$$

where $\mathbf{F} \in \mathbb{R}^{s q \times l}$ denotes the evaluation of the basis functions of $Q_{h}$ at quadrature points $x_{i}, i=$ $1, \ldots, q$, and $\mathbf{E} \in \mathbb{R}^{s q \times m}$ the evaluation of the strain components for the basis functions in $W_{h}$ at the same quadrature points. Note that each quadrature point appears $s$ times, once for each component of the stress tensor. The components in $\boldsymbol{\omega}=\left(\omega_{1}, \ldots, \omega_{1}, \omega_{2}, \ldots, \omega_{2}, \ldots, \omega_{q}, \ldots, \omega_{q}\right)^{T} \in \mathbb{R}^{s q}$ are products of quadrature weights with the Jacobi determinants. Note that each value $\omega_{j}$ occurs $s$ times in $\boldsymbol{\omega}$. The entries in the vector $\varrho(\underline{\boldsymbol{u}})=\left(\varrho_{1}(\underline{\boldsymbol{u}}), \ldots, \varrho_{1}(\underline{\boldsymbol{u}}), \varrho_{2}(\underline{\boldsymbol{u}}) \ldots \varrho_{q}(\underline{\boldsymbol{u}})\right)^{T} \in \mathbb{R}^{s q}$ are given by

$$
\varrho_{j}(\underline{\boldsymbol{u}})=v \max \left(0,1-g\left\|\mathbf{E}_{j} \underline{\boldsymbol{u}}\right\|^{-1}\right) \text { for } j=1, \ldots, q .
$$

Here, $\mathbf{E}_{j}$ are the rows of $\mathbf{E}$ corresponding to the $j$ th quadrature point, and $\left\|\mathbf{E}_{j} \underline{\boldsymbol{u}}\right\|=\left(\underline{\underline{\boldsymbol{u}}} \underline{\mathbf{E}}_{j}^{T} \mathbf{E}_{j} \underline{\boldsymbol{u}}\right)^{1 / 2}$. Note that in the discretized form, the nonlinearity comes into play at the quadrature points.

Next, we study the nonlinear system (30). First, note that using the notation introduced above, the mass matrix $\mathbf{N}$ is given by $\mathbf{N}=\mathbf{F}^{T} \operatorname{diag}(\omega) \mathbf{F}$. Since $\mathbf{N}$ is invertible, $\underline{\boldsymbol{\tau}}$ can be eliminated from 
(30), which results in the reduced system

$$
\left[\begin{array}{cc}
\mathbf{A}+\mathbf{L}(\underline{\boldsymbol{u}}) & \mathbf{B}^{T} \\
\mathbf{B} & \mathbf{0}
\end{array}\right]\left[\begin{array}{l}
\underline{\boldsymbol{u}} \\
\boldsymbol{p}
\end{array}\right]=\left[\begin{array}{c}
\underline{\boldsymbol{f}} \\
\mathbf{0}
\end{array}\right] \quad \text { with } \mathbf{L}(\underline{\boldsymbol{u}})=\mathbf{E}^{T} \operatorname{diag}(\boldsymbol{\omega}) \mathbf{P} \operatorname{diag}(\varrho(\underline{\boldsymbol{u}})) \mathbf{E} .
$$

Here, $\mathbf{P}=\mathbf{F}\left(\mathbf{F}^{T} \operatorname{diag}(\boldsymbol{\omega}) \mathbf{F}\right)^{-1} \mathbf{F}^{T} \operatorname{diag}(\boldsymbol{\omega})$ is the projection onto the space spanned by columns of $\mathbf{F}$. Note that, in general (33) does not represent the first-order necessary conditions of an optimization problem. However, if $\mathbf{P}=\operatorname{diag}\left(p_{1}, \ldots, p_{1}, p_{2}, \ldots, p_{q}\right) \in \mathbb{R}^{s q \times s q}$ is diagonal, the matrix $\mathbf{L}(\underline{\boldsymbol{u}})$ is symmetric and positive definite for all $\underline{\boldsymbol{u}} \in \mathbb{R}^{m}$ and thus the solution of (33) is also characterized as minimizer of a strictly convex optimization problem with equality constraints, namely

$$
\min _{\mathbf{B} \underline{\boldsymbol{u}}=0} \frac{1}{2} \underline{\boldsymbol{u}}^{T} \mathbf{A} \underline{\boldsymbol{u}}+\sum_{j=1}^{q} \nu \omega_{j} p_{j} \max \left(0,\left\|\mathbf{E}_{j} \underline{\boldsymbol{u}}\right\|-g\right)^{2} .
$$

As a consequence, the system (33) has a unique solution. To summarize:

LEMMA 5.1 If the velocity-pressure finite element discretization satisfies the discrete inf-sup condition and $\mathbf{P}$ is diagonal, the nonlinear system (30) has a unique solution $\left(\underline{\boldsymbol{u}}^{\star}, \underline{\boldsymbol{p}}^{\star}, \underline{\boldsymbol{\tau}}^{\star}\right)$.

An example for a discretization of $Q_{h}$ that results in a diagonal matrix $\mathbf{P}$ are piecewise discontinuous constants - this discretization is used for the numerical results presented in Section 6. Also, quadrature rules that use the same points for interpolation and quadrature (often, LegendreGauss-Lobatto points are used in this context) result in diagonal $\mathbf{N}$ and thus a diagonal matrix $\mathbf{P}$.

We now turn to the discretization for the time-dependent shear thickening model (12). While a variety of methods for the time discretization of (12) can be used, we choose the implicit Euler method, for simplicity. Finite elements as described above are used for the space discretization. For the time discretization, we split $[0, T]$ into $K$ equal-size intervals of length $\Delta t=T / K$ and denote, for $k=0, \ldots, K$, the discrete solution at time $t^{k}:=k \Delta t$ by $\left(\boldsymbol{u}_{h}^{k}, p_{h}^{k}, \boldsymbol{\tau}_{h}^{k}\right)$. Then, the discretized problem amounts to solving, for each $k=1, \ldots, K$ the system

$$
\begin{aligned}
& \frac{1}{\Delta t}\left(\boldsymbol{u}_{h}^{k}-\boldsymbol{u}_{h}^{k-1}, \boldsymbol{v}_{h}\right)+\mu\left(\varepsilon \boldsymbol{u}_{h}^{k}, \varepsilon \boldsymbol{v}_{h}\right)+\left(\boldsymbol{\tau}_{h}^{k}, \varepsilon \boldsymbol{v}_{h}\right) \\
& \quad+\left(p_{h}^{k}, \nabla \cdot \boldsymbol{v}_{h}\right)+\left(q_{h}^{k}, \nabla \cdot \boldsymbol{u}_{h}^{k}\right)-\left(\boldsymbol{f}^{k}, \boldsymbol{v}_{h}\right)=0 \text { for all }\left(\boldsymbol{v}_{h}, q_{h}\right) \in W_{h} \times P_{h}, \\
& \quad v\left(\max \left(0,1-g\left|\varepsilon \boldsymbol{u}_{h}^{k}\right|^{-1}\right) \varepsilon \boldsymbol{u}_{h}^{k}, \boldsymbol{\varphi}_{h}\right)-\left(\boldsymbol{\tau}_{h}^{k}, \boldsymbol{\varphi}_{h}\right)=0 \text { for all } \boldsymbol{\varphi}_{h} \in Q_{h} .
\end{aligned}
$$

Above, $\boldsymbol{f}^{k}=\boldsymbol{f}\left(t^{k}\right)$ and $\boldsymbol{u}_{h}^{0}=\boldsymbol{u}_{0}$ is the initial condition. Note that no initial condition for the pressure and the dual tensor are needed. Using quadrature to approximate the integrals in (34), and denoting by $\left(\underline{\boldsymbol{u}}^{k}, \underline{\boldsymbol{p}}^{k}, \underline{\boldsymbol{\tau}}^{k}\right)$ the finite element coefficients corresponding to $\left(\boldsymbol{u}_{h}^{k}, p_{h}^{k}, \boldsymbol{\tau}_{h}^{k}\right)$ for $k=$ $0, \ldots, K$, the nonlinear algebraic system corresponding to (34) is:

$$
\left[\begin{array}{ccc}
\mathbf{A}+\frac{1}{\Delta t} \mathbf{M} & \mathbf{B}^{T} & \mathbf{G}^{T} \\
\mathbf{B} & \mathbf{0} & \mathbf{0} \\
\tilde{\mathbf{G}}(\underline{\boldsymbol{u}}) & \mathbf{0} & -\mathbf{N}
\end{array}\right]\left[\begin{array}{c}
\underline{\boldsymbol{u}}^{k} \\
\underline{\boldsymbol{p}}^{k} \\
\underline{\boldsymbol{\tau}}^{k}
\end{array}\right]=\left[\begin{array}{c}
\underline{\boldsymbol{f}}^{k}+\frac{1}{\Delta t} \mathbf{M} \underline{\boldsymbol{u}}^{k-1} \\
\mathbf{0} \\
\mathbf{0}
\end{array}\right] .
$$

This system is similar to the nonlinear system for steady shear thickening flow (30). Due to the max-function involved in these systems, they are not differentiable and we cannot apply the classical Newton's method for their solution. However, we can employ a Newton method that uses a generalized differentiability concept to solve (30), as shown in the next section. 


\subsection{Generalized Newton algorithm}

We first compute derivatives of the nonlinear term $\tilde{\mathbf{G}}(\underline{\boldsymbol{u}})$ in (30). Generalized (or semismooth) derivatives in finite dimensions are well studied in the literature [21, 31, 32]. It can be shown that the max-function is differentiable in this generalized sense. A particular choice of generalized derivative of the max-function to compute the derivative of $\varrho_{j}(\underline{\boldsymbol{u}})$ (see (32)) in a direction $\underline{\tilde{\boldsymbol{u}}} \in \mathbb{R}^{m}$ results in

$$
\varrho_{j}^{\prime}(\underline{\boldsymbol{u}})(\underline{\tilde{\boldsymbol{u}}})=v \chi_{j} g\left\|\mathbf{E}_{j} \underline{\boldsymbol{u}}\right\|^{-3} \underline{\boldsymbol{u}}^{T} \mathbf{E}_{j}^{T} \mathbf{E}_{j} \underline{\tilde{\boldsymbol{u}}} \quad \text { with } \chi_{j}= \begin{cases}1 & \text { if }\left\|\mathbf{E}_{j} \underline{\boldsymbol{u}}\right\| \geqslant g, \\ 0 & \text { else. }\end{cases}
$$

Thus, we obtain the generalized derivative of $\tilde{\mathbf{G}}(\underline{\boldsymbol{u}})$

$$
\tilde{\mathbf{G}}^{\prime}(\underline{\boldsymbol{u}})(\underline{\tilde{\boldsymbol{u}}})=\mathbf{F}^{T} \operatorname{diag}(\boldsymbol{\omega}) \operatorname{Diag}\left(\mathbf{R}_{j}(\underline{\boldsymbol{u}})\right) \mathbf{E} \underline{\tilde{\boldsymbol{u}}},
$$

where $\operatorname{Diag}\left(\mathbf{R}_{j}(\underline{\boldsymbol{u}})\right) \in \mathbb{R}^{q s \times q s}$ is a block diagonal matrix, with blocks

$$
\mathbf{R}_{j}(\underline{\boldsymbol{u}})=v \chi_{j}\left[\left(1-g\left\|\mathbf{E}_{j} \underline{\boldsymbol{u}}\right\|^{-1}\right) \mathbf{I}_{s}+g\left\|\mathbf{E}_{j} \underline{\boldsymbol{u}}\right\|^{-3} \mathbf{E}_{j} \underline{\boldsymbol{u}} \underline{\boldsymbol{u}}^{T} \mathbf{E}_{j}^{T}\right] \in \mathbb{R}^{s \times s},
$$

for $j=1, \ldots, q$, where $\mathbf{I}_{s} \in \mathbb{R}^{s \times s}$ denotes the identity matrix. Above, the identity

$$
\left(\underline{\boldsymbol{u}}^{T} \mathbf{E}_{j}^{T} \mathbf{E}_{j} \underline{\tilde{u}}\right) \mathbf{E}_{j} \underline{\boldsymbol{u}}=\left(\mathbf{E}_{j} \underline{\boldsymbol{u}} \underline{\boldsymbol{u}}^{T} \mathbf{E}_{j}^{T}\right) \mathbf{E}_{j} \underline{\tilde{u}}
$$

is used. We can now give the generalized Newton step for (30). Given the $k$ th iterate $\left(\underline{\boldsymbol{u}}^{k}, \underline{\boldsymbol{p}}^{k}, \underline{\boldsymbol{\tau}}^{k}\right)$, the Newton update $(\underline{\hat{\boldsymbol{u}}}, \hat{\boldsymbol{p}}, \underline{\hat{\boldsymbol{\tau}}})$ is computed by solving

$$
\left[\begin{array}{ccc}
\mathbf{A} & \mathbf{B}^{T} & \mathbf{G}^{T} \\
\mathbf{B} & \mathbf{0} & \mathbf{0} \\
\tilde{\mathbf{G}}^{\prime}\left(\underline{\boldsymbol{u}}^{k}\right) & \mathbf{0} & -\mathbf{N}
\end{array}\right]\left[\begin{array}{l}
\frac{\hat{\boldsymbol{u}}}{\hat{\hat{p}}} \\
\frac{\hat{\hat{\tau}}}{\underline{\boldsymbol{c}}}
\end{array}\right]=\left[\begin{array}{c}
\underline{\boldsymbol{f}}-\mathbf{A} \underline{\boldsymbol{u}}^{k}-\mathbf{B}^{T} \boldsymbol{p}^{k}-\mathbf{G}^{T} \underline{\boldsymbol{\tau}}^{k} \\
-\mathbf{B} \underline{\boldsymbol{u}}^{k} \\
-\tilde{\mathbf{G}}\left(\underline{\boldsymbol{u}}^{k}\right)+\mathbf{N} \underline{\boldsymbol{\tau}}^{k}
\end{array}\right]
$$

and the next Newton iteration obtained from

$$
\left(\underline{\boldsymbol{u}}^{k+1}, \underline{\boldsymbol{p}}^{k+1}, \underline{\boldsymbol{\tau}}^{k+1}\right):=\left(\underline{\boldsymbol{u}}^{k}, \underline{\boldsymbol{p}}^{k}, \underline{\boldsymbol{\tau}}^{k}\right)+(\underline{\hat{\boldsymbol{u}}}, \underline{\hat{\boldsymbol{p}}}, \underline{\hat{\boldsymbol{\tau}}}) .
$$

Due to the generalized differentiability of (30), we obtain the following convergence result for the Newton method based on the iteration rule (36), where we again restrict ourselves to discretizations leading to a diagonal matrix $\mathbf{P}$.

LEMMA 5.2 If the velocity-pressure finite element discretization satisfies the discrete inf-sup condition and $\mathbf{P}$ is diagonal, the (generalized) Newton matrix in (36a) is invertible for every $\underline{\boldsymbol{u}}^{k} \in \mathbb{R}^{m}$, and the inverse matrices are bounded independently of $\underline{\boldsymbol{u}}^{k}$.

Proof. As in Section 5, we eliminate the variable $\underline{\hat{\boldsymbol{\tau}}}$ from (36a) and obtain

$$
\left[\begin{array}{cc}
\mathbf{A}+\mathbf{L}^{\prime}\left(\underline{\boldsymbol{u}}^{k}\right) & \mathbf{B}^{T} \\
\mathbf{B} & \mathbf{0}
\end{array}\right][\underline{\underline{\hat{\boldsymbol{u}}}} \underline{\hat{\boldsymbol{p}}}]=\left[\underline{\boldsymbol{f}}-\mathbf{A} \underline{\boldsymbol{u}}^{k}-\mathbf{B}^{T} \underline{\boldsymbol{p}^{k}}-\mathbf{G}^{T} \mathbf{N}^{-1} \tilde{\mathbf{G}}\left(\underline{\boldsymbol{u}}^{k}\right)\right]
$$

with $\mathbf{L}^{\prime}(\underline{\boldsymbol{u}})=\mathbf{E}^{T} \operatorname{diag}(\boldsymbol{\omega}) \mathbf{P D i a g}\left(\mathbf{R}_{j}(\underline{\boldsymbol{u}})\right) \mathbf{E}$. If $\mathbf{P}$ is $\operatorname{diagonal}$, then $\mathbf{L}^{\prime}\left(\underline{\boldsymbol{u}}^{k}\right)$ is symmetric and positive semidefinite and thus the system (37) has a unique solution. Moreover, as A is positive definite, the inf-sup constant can be chosen independently of $\underline{\boldsymbol{u}}^{k}$ and, hence, the inverse matrices are bounded. 
Convergence results for generalized Newton methods in finite-dimensional spaces [21, 32] can be applied, and thus the following convergence result is obtained.

THeORem 5.3 Let the initialization $\left(\underline{\boldsymbol{u}}^{0}, \boldsymbol{p}^{0}, \underline{\boldsymbol{\tau}}^{0}\right)$ be sufficiently close to $\left(\underline{\boldsymbol{u}}^{\star}, \boldsymbol{p}^{\star}, \underline{\boldsymbol{\tau}}^{\star}\right)$. Then the Newton iterates $\left(\underline{\boldsymbol{u}}^{k}, \underline{\boldsymbol{p}}^{k}, \underline{\boldsymbol{\tau}}^{k}\right), k=1,2, \ldots$ computed from (36) converge to $\left(\underline{\boldsymbol{u}}^{\star}, \underline{\boldsymbol{p}}^{\star}, \underline{\boldsymbol{\tau}}^{\star}\right)$ at a superlinear rate.

A generalized Newton method as described above also applies for the solution of (35), and a fast local convergence result analogously to Theorem 5.3 holds for the application of the generalized Newton method to solve the nonlinear system in each time step. Since the Newton iteration for a time step can be initialized with the solution from the previous step, only few iterations are required in each time step thanks to the fast local convergence of the method. In the next section, we apply the above algorithms for the solution of steady and unsteady flow problems with shear thickening rheology.

\section{Numerical results}

The purpose of this section is, first, to study the discretization and the solution methods proposed in Section 5, and, second, to examine the flow behavior and the free boundary resulting from our shear thickening flow model, and to study how this behavior compares with experiments from the literature. We use dimensional variables in SI units, i.e., $\mathrm{m} / \mathrm{s}$ for the velocity, $1 / \mathrm{s}$ for the strain rate, $\mathrm{Pa}$ for the stress, and Pa.s for the viscosity. The linear Stokes systems arising in each step of the Newton method are solved with a direct solver. Note, however, that the linearized problems are Stokes problems with varying viscosity, for which efficient iterative solvers are available [6, 9, 20, 30]. We use the lowest-order Taylor-Hood pair to discretize velocity and pressure, i.e., continuous quadratic elements for each velocity component and continuous linear elements for the pressure. Each component of $\boldsymbol{\tau}$ is discretized using element-wise constants. For steady-state problems, the Newton iteration is initialized with the solution of the linear Stokes equation without shear thickening. For time-dependent problems, the solution from the previous time step is used as initialization. The Newton iteration is terminated when the $L_{2}$-norm of the nonlinear residual is decreased by 10 orders of magnitude.

\subsection{Problem I: Steady channel flow around an obstacle}

We consider the steady flow through a channel, which contains an obstacle. The channel has a length of $0.4 \mathrm{~m}$ and a height of $0.2 \mathrm{~m}$. It is assumed to either be of large depth, or axially symmetric - in both cases, the flow can be modeled in two dimensions. The flow is driven by a parabolic inflow boundary condition with maximal velocity $u_{0}$, i.e., $\boldsymbol{u}=\left(u_{0}\left(100 y^{2}-1\right), 0\right)^{T}$ for the left boundary. On the right side, a traction-free outflow boundary condition is used. All other boundaries, including the obstacle, satisfy no-flow (i.e., homogeneous Dirichlet) conditions. The obstacles we consider are centered at the origin of the coordinate system. Since they are symmetric with respect to the channel's centerline, it suffices to compute the two-dimensional flow in half of the channel. Thus, our computational domain is $\Omega=[-0.2,0.2] \times[0,0.1]$, and we use symmetry boundary conditions $(\boldsymbol{u} \cdot \boldsymbol{n}=0$ and free slip in tangential direction) for the bottom boundary $[-0.2,0.2] \times\{0\}$.

6.1.1 Two-dimensional flow around circular and square-shaped obstacles. Here, the obstacle is either a sphere with radius 0.025 , or a square with edge length 0.04 ; both are centered at the origin. 
We first study convergence properties of our algorithm, and then focus on the qualitative behavior of shear thickening flow, and on the free boundary.

Convergence rate. We first study the convergence of the proposed generalized Newton method. For that purpose, we use the channel with circular obstacle and a maximal inflow rate of $u_{0}=0.03$. In Figure 2 (left), the nonlinear residual in each Newton step is plotted. Note the fast convergence close to the solution, as is typical for Newton-type methods (compare with Theorem 5.3). The residual as a function of the iteration number behaves similar for all other tested values of $u_{0}$.

Dependence of the number of iterations on the mesh and v. On the right hand side in Figure 2, we report on the number of Newton iterations needed for convergence for different discretizations and values of $v$. Note that we observe a mesh-independent behavior for fixed $v$, i.e., the number of iterations is stable with respect to mesh refinement. However, increasing $v$ leads to a larger number of Newton iteration. This suggest that the simulation of shear thickening fluids with very different viscosities could be accelerated using continuation with respect to $v$. However, note that the limit case of diverging viscosity $v \rightarrow \infty$ might be more of theoretical than of practical interest, since most experiments with colloidal dispersions report a finite increase in viscosity ranging from one to several orders of magnitude $[5,12,29]$.

Comparison with linear Stokes flow. In Figure 3, we compare the streamlines obtained with a linear Stokes model with those for a shear thickening fluid. As can be observed, the flow lines concentrate further away from the boundary layers for shear thickening flow. Note that due to the inflow boundary condition, in both cases the overall flow rate through the channel is the same, while the qualitative behavior of the flow differs.

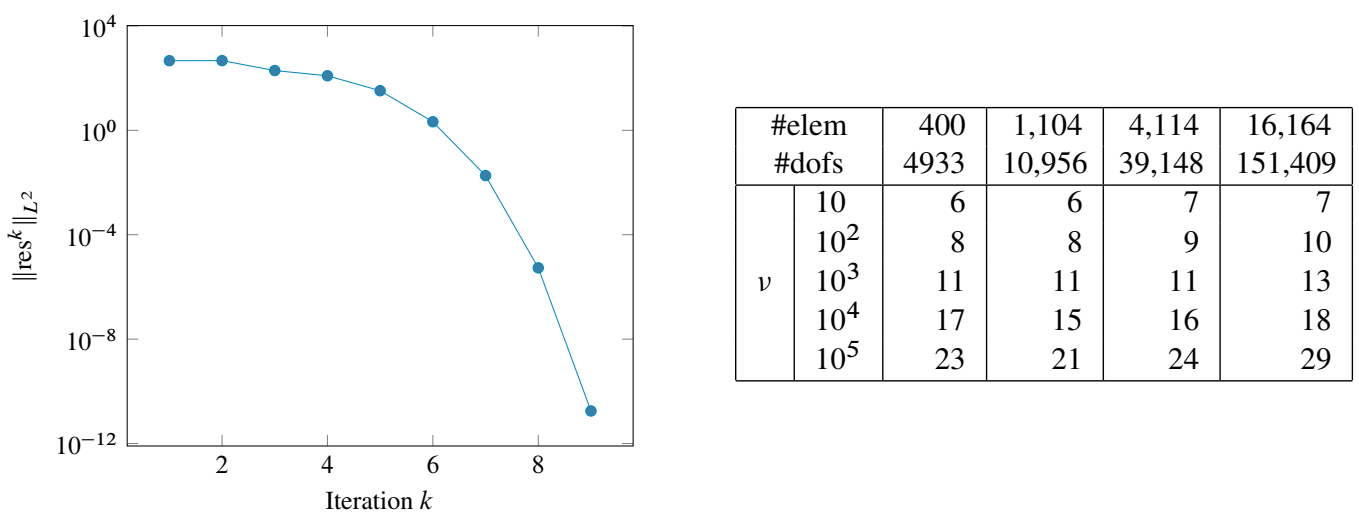

FIG. 2. Problem I: Convergence of generalized Newton method for shear thickening constitutive law for a simulation with $g=1$ and $u_{0}=0.03$. On the left, we plot the $L^{2}$-norm of the nonlinear residual in the $k$-th iteration, $\|$ res ${ }^{k} \|$, for $v=100$ on a 4,114 element mesh. The table on the right displays the number of Newton iterations required to decrease the residual by 10 orders of magnitude. Shown are the iteration numbers for meshes with different numbers of finite elements (\#elem) and unknowns in the Stokes system (\#dofs) for various values of $v$. 


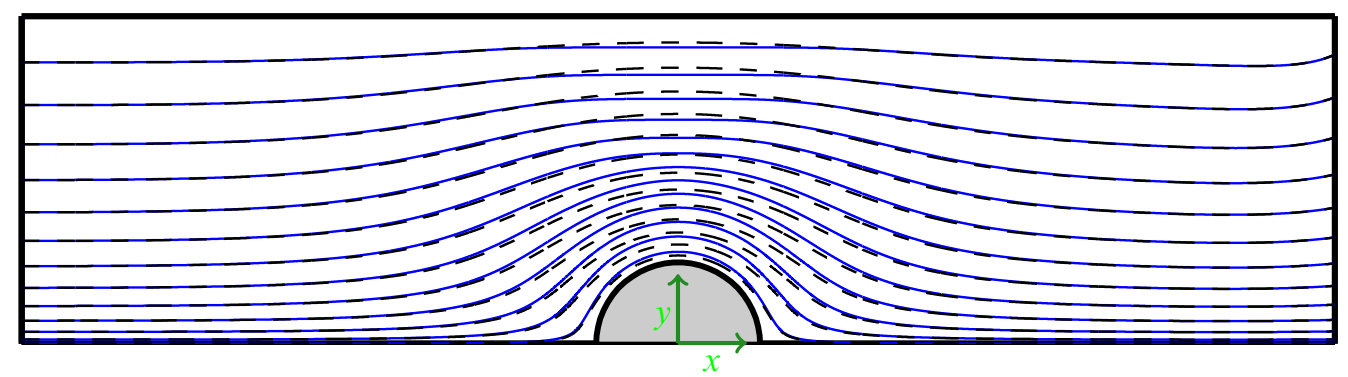

FIG. 3. Problem I: Streamlines in upper half of channel with a spherical obstacle (gray) for parabolic inflow with maximal velocity $u_{0}=0.035$. Shown are the streamlines for Stokes flow without shear thickening (dashed lines) and with discontinuous shear thickening using $g=1$ and $v=100$ (solid lines). Indicated is also the coordinate system.
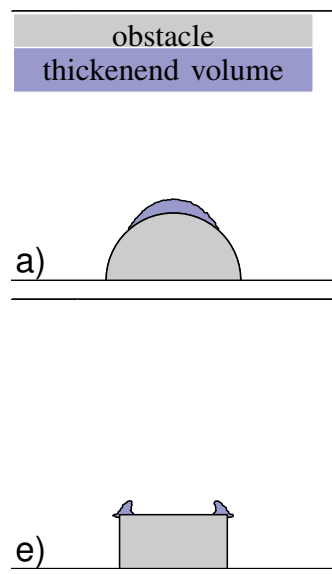
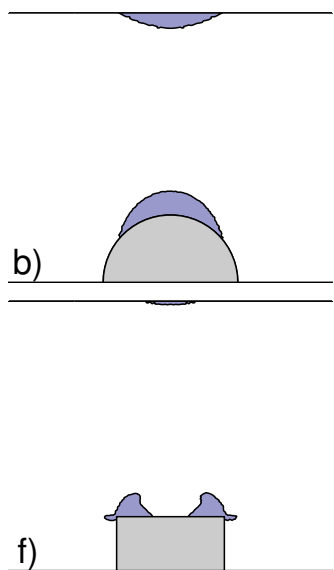
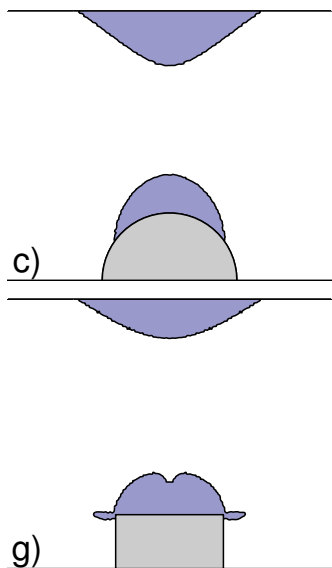
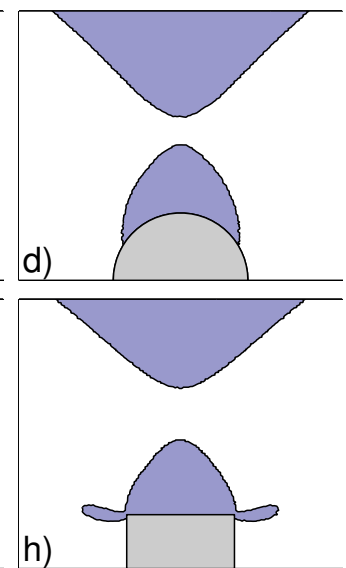

FIG. 4. Problem I: Comparison of free boundaries arising in the discontinuous shear thickening flow model for the circular obstacle $(a$ to $d)$ and square-shaped obstacle ( $e$ to $h)$. The columns correspond to the different inflow velocities $u_{0}=0.02(a$ and $e), u_{0}=0.025(b$ and $f), u_{0}=0.03(c$ and $g)$ and $u_{0}=0.035(d$ and $h)$. Shown is the part $[-0.06,0.06] \times[0,0.1]$ of the channel; both, the obstacle and the shear thickened volume are shown.

Free boundaries. We define the thickened volume as all points where $|\varepsilon \boldsymbol{u}|$ is at or above the threshold $g$, i.e., as $\{x \in \Omega:|\varepsilon \boldsymbol{u}(x)| \geqslant g\}$. We call the boundary of this set, i.e., $\{x \in \Omega$ : $|\mathcal{E} \boldsymbol{u}(x)|=g\}$ the free boundary. In Figure 4, we show this free boundary for different inflow velocities $u_{0}$ for the circular and the square-shaped obstacles. As to be expected, the amount of shear thickened volume grows with the flow rate, and thus the shear strain rate increases. In our example problems, we observe a monotone growth of the thickened volume. The shear thickening regions are close-to-symmetric with respect to the $y$-axis-potential asymmetry can only be caused by the different inflow and outflow boundary conditions. For the square-shaped obstacle and low flow rates, the thickened volume is concentrated around the corners since these re-entrant corners are areas with high strain rates. 

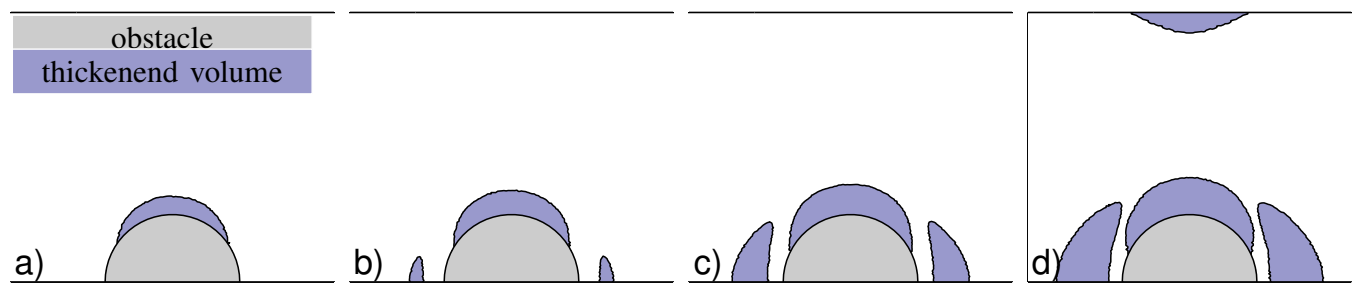

FIG. 5. Problem I, axially symmetric flow: Shown is the part $[-0.06,0.06] \times[0,0.1]$ of the channel; both, the obstacle and the shear thickened volume are shown.

6.1.2 Axially symmetric flow around a sphere. Cylindrical coordinates for the Stokes equation are used to model axially symmetric flow. The free boundaries of the shear hardened regions that occur for different inflow velocities are shown in Figure 5. Comparing with the upper row in Figure 4, less thickening occurs for the same inflow velocity in the axially symmetric problem, since the strain rates caused by a spherical obstacle are smaller than those caused by a circular rod. Observe the lens-shaped thickened volume at the front and top of the spherical obstacle.

\subsection{Problem II: Flow driven by a moving obstacle}

The geometry in this problem coincides with the axially symmetric case in Problem I (Section 6.1.2), but we assume no-flow boundary conditions on the whole boundary. The flow is initially at rest (i.e., $\left.\boldsymbol{u}_{0}=0\right)$ and is driven only by the motion of the sphere, whose center $\boldsymbol{c}=\left(c_{1}, c_{2}\right)$ follows the time-dependent trajectory

$$
c_{1}(t)=u_{0}\left(\min (t, 0.5)^{2}+\max (0, t-0.5)\right), \quad c_{2}(t)=0 \quad \text { for } t \in[0, T] .
$$

As before, the sphere has the radius $r=0.025$ and the rotational component of its motion is zero. Note that the sphere's velocity grows linearly until it reaches the velocity $u_{0}>0$ at $t=0.5$. We stop the simulation at $T:=(0.2-r) / u_{0}+0.25$ when the sphere touches the right boundary of the domain. The setup of this problem is motivated by the experimental study in [28], in which a solid body is pushed through a concentrated cornstarch suspension towards a wall made of molding clay. In this experiment, the thickened volume at the leading side of the sphere causes an imprint in the clay.

A fictitious domain method with distributed Lagrange multipliers $[18,19]$ is used to simulate the interaction between the fluid and the rigid sphere. In this method, the rigid sphere is thought of being filled with the surrounding fluid, and distributed Lagrange multipliers are used to impose the prescribed rigid motion of the sphere. This computation can be performed on a fixed mesh, which is a major advantage of the fictitious domain method compared to methods that are based on mapped meshes or require re-meshing. Note that our problem is one-way coupled, i.e., only the force acting on the fluid due to the prescribed trajectory of the rigid sphere must be considered. The fictitious domain method also applies to problems with fully coupled fluid-solid interaction, as, for instance, the settling of a rigid body in a shear thickening fluid, [38].

To sketch the application of the fictitious domain method to our problem, we denote the region covered by the rigid sphere at time $t$ by $\bar{\Omega}(t) \subset \Omega$ and introduce a finite element space $R_{h}\left(t^{k}\right) \subset$ $H^{1}\left(\bar{\Omega}\left(t^{k}\right)\right)^{s}$, which is defined over $\bar{\Omega}(t)$. In each time step, the finite element discretized system 
(34) for the flow at time $t^{k}$ is augmented by the fictitious domain constraint

$$
\left(\boldsymbol{u}^{k}-\boldsymbol{c}^{\prime}\left(t^{k}\right), \boldsymbol{r}_{h}\right)_{\bar{\Omega}\left(t^{k}\right)}=0 \text { for all } \boldsymbol{r}_{h} \in R_{h}\left(t^{k}\right)
$$

where $(\cdot, \cdot)_{\bar{\Omega}\left(t^{k}\right)}$ denotes a scalar product over $\bar{\Omega}\left(t^{k}\right)$ and the velocity of the sphere at time $t$ is $c^{\prime}(t)$, with $c$ as defined in (38). From the continuous standpoint, the $H^{1}\left(\Omega\left(t^{k}\right)\right)$ scalar product should be used for $(\cdot, \cdot) \bar{\Omega}_{\left(t^{k}\right)}$. However, for the discretized problem it is a valid choice (and common practice) to use a discrete inner product that approximates the weaker $L^{2}\left(\Omega\left(t^{k}\right)\right)$ inner product for (39). We use the sum of the pointwise nodal values corresponding to the velocity degrees of freedom inside $\bar{\Omega}(t)$, as well as points on the surface $\partial \bar{\Omega}(t)$ for the discrete inner product, which amounts to a collocation method for the fictitious domain constraints, [18, 19]. Figure 6 shows an example mesh together with the points at which the fictitious domain constraints are enforced.

To compute the flow solution at a time step $t^{k}$, both the incompressibility constraint and the fictitious domain constraint (39) must be taken into account, which can be tricky. As a remedy, we use an operator splitting method, in which the incompressibility condition and the fictitious domain condition are neglected alternately in fractional time steps. Given iterates $\left(\boldsymbol{u}_{h}^{k-1}, p_{h}^{k-1}, \boldsymbol{\tau}_{h}^{k-1}\right)$, this amounts to first solving the fictitious domain problem

$$
\begin{aligned}
\frac{1}{\delta t}\left(\boldsymbol{u}_{h}^{k-1 / 2}-\boldsymbol{u}_{h}^{k-1}, \boldsymbol{v}_{h}\right)+\alpha \mu\left(\varepsilon \boldsymbol{u}_{h}^{k-1 / 2}, \mathcal{E} \boldsymbol{v}_{h}\right) & \\
+\left(\lambda_{h}^{k-1 / 2}, \boldsymbol{u}^{k-1 / 2}\right)_{\bar{\Omega}\left(t^{k}\right)}+\left(p_{h}^{k-1}, \nabla \cdot \boldsymbol{u}_{h}^{k-1 / 2}\right) & =0 \text { for all } \boldsymbol{v}_{h} \in W_{h}, \\
\left(\boldsymbol{u}^{k-1 / 2}-\boldsymbol{c}^{\prime}\left(t^{k}\right), \boldsymbol{r}_{h}\right)_{\bar{\Omega}\left(t^{k}\right)} & =0 \text { for all } \boldsymbol{r}_{h} \in R_{h}\left(t^{k}\right),
\end{aligned}
$$

for $\left(\boldsymbol{u}^{k-1 / 2}, \lambda^{k-1 / 2}\right) \in W_{h} \times R_{h}\left(t^{k}\right)$, where $\lambda^{k-1 / 2}$ is the Lagrange multiplier for the fictitious domain constraint, and $0 \leqslant \alpha \leqslant 1$ (below, $\alpha=0.5$ is used). Then, a shear thickening flow problem

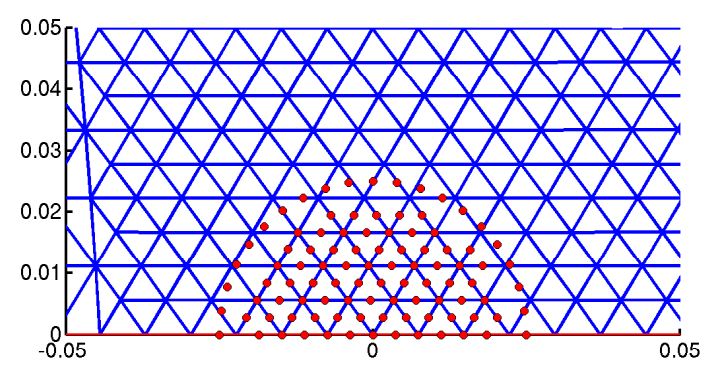

FIG. 6. Problem II: Computational mesh and points covering the fictitious domain $\bar{\Omega}(0)$ at $t=0$. Shown is the region $[-0.05,0.05] \times[0,0.05] \subset \Omega$. The mesh used in our computations is finer than this mesh, and thus also uses a larger number of points to cover the fictitious domain. To avoid an over-constrained problem, the fictitious domain constraint is not imposed at mesh points close to $\partial \bar{\Omega}(0),[19]$. 
similar to (34) is solved for $\left(\boldsymbol{u}_{h}^{k}, p_{h}^{k}, \boldsymbol{\tau}_{h}^{k}\right)$ :

$$
\begin{aligned}
\frac{1}{\delta t}\left(\boldsymbol{u}_{h}^{k}-\boldsymbol{u}_{h}^{k-1 / 2}, \boldsymbol{v}_{h}\right)+(1-\alpha) \mu\left(\mathcal{E} \boldsymbol{u}_{h}^{k}, \mathcal{E} \boldsymbol{v}_{h}\right) & +\left(\boldsymbol{\tau}_{h}^{k}, \mathcal{E} \boldsymbol{v}_{h}\right)+\left(\lambda^{k-1 / 2}, \boldsymbol{u}^{k}\right) \bar{\Omega}\left(t^{k}\right) \\
+\left(p_{h}^{k}, \nabla \cdot \boldsymbol{v}_{h}\right)+\left(q_{h}^{k}, \nabla \cdot \boldsymbol{u}_{h}^{k}\right) & =0 \text { for all }\left(\boldsymbol{v}_{h}, q_{h}\right) \in W_{h} \times P_{h}, \\
v\left(\max \left(0,1-g\left|\mathcal{E} \boldsymbol{u}_{h}^{k}\right|^{-1}\right) \varepsilon \boldsymbol{u}_{h}^{k}, \boldsymbol{\varphi}_{h}\right)-\left(\boldsymbol{\tau}_{h}^{k}, \boldsymbol{\varphi}_{h}\right) & =0 \text { for all } \boldsymbol{\varphi}_{h} \in Q_{h} .
\end{aligned}
$$

The error due to the operator splitting is mitigated by using the pressure $p_{h}^{k-1}$ from the previous time step in (40a) and the fictitious domain Lagrange multiplier $\lambda^{k-1 / 2}$ in (40b). Additionally, the splitting error can be controlled by choosing a small time step.

We use 250 time steps and a mesh with 26,609 triangular elements for our computations. The mesh is refined around the trajectory of the rigid sphere to ensure that the fictitious domain and the free boundary of the thickened volume are sufficiently well resolved. Figure 7 shows snapshots of the solution for $u_{0}=0.03$ in (38), i.e., after the start-up phase (for $t<0.5$ ), the sphere moves with a constant velocity of 0.03 to the right. First, note that in Figure 7 (a), where the sphere is still far away from the right boundary, the thickened volume resembles the steady state solution for an axially symmetric channel with $u_{0}=0.03$ (left image in Figure 5). Figures 7 (b), (c), (d) and (e) show that additional solidification occurs at the leading side of the sphere caused by the increased strain rate due to the redirection of the flow at the right boundary of $\Omega$. This thickened volume transmits force from the sphere to the right boundary as shown in the right column of Figure 7, where the outward normal traction acting on the right boundary is shown. Such a force transmission is also found in an experimental study with a cornstarch-water dispersion [28], and it is a particular property of fluids that yield thickening at high shear rates.

\subsection{Problem III: A solid rod impacting the fluid surface}

Next, we study the time evolution of the flow due to an applied surface force. Our problem setup is motivated by a recent experiment reported in [40], in which a circular rod plunges into a container filled with cornstarch. The authors find impact-activated solidification below and around the rod's impact zone. They argue that the main source of energy dissipation is that this jammed zone causes a large blob of material to move down with it.

To numerically model a similar experiment, we consider half of a slice through an axially symmetric setup similar to the one described above. We use the computational domain $\Omega=$ $[-0.1,0.1] \times[0,0.25]$, the symmetry axis $x=0$, final time $T=0.005$ and the initial condition $\boldsymbol{u}_{0}=0$. Dirichlet boundary conditions for the bottom and right, and a symmetry condition for the left boundary are imposed. The upper boundary is traction-free in tangential direction, and the forcing in the normal direction is given by

$$
\boldsymbol{\sigma n} \cdot \boldsymbol{n}=\left\{\begin{array}{cl}
-50 & \text { for } x \leqslant 0.01 \text { and } t \leqslant 0.6 T \\
0 & \text { else }
\end{array}\right.
$$

where $\boldsymbol{n}$ denotes the outward normal unit vector. Moreover, we use $v=10^{3}$ and $g=1$.

Our simulation uses a triangular mesh with 4,262 elements, which is refined around the area where the boundary force is applied. Moreover, 200 time steps are used for the time discretization. The number of Newton iterations needed to solve the nonlinear system in each time step is shown 
a)

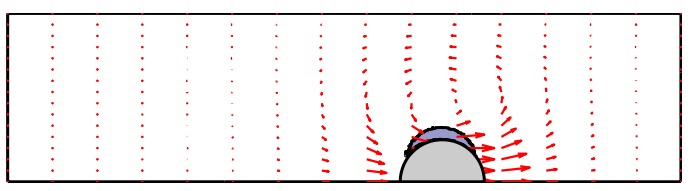

b)

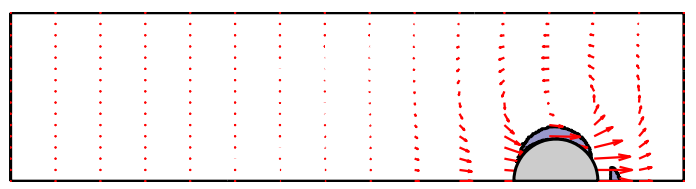

c)

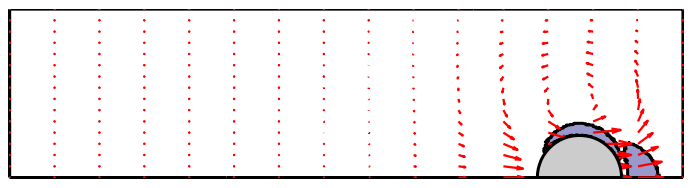

d)

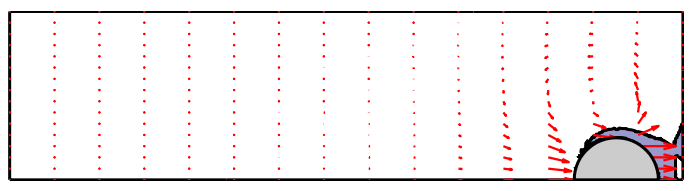

e)

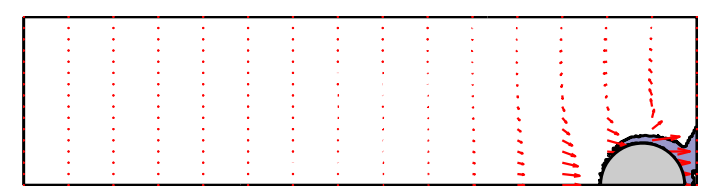

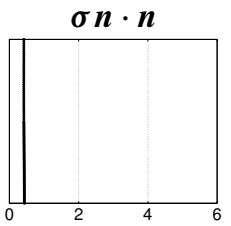
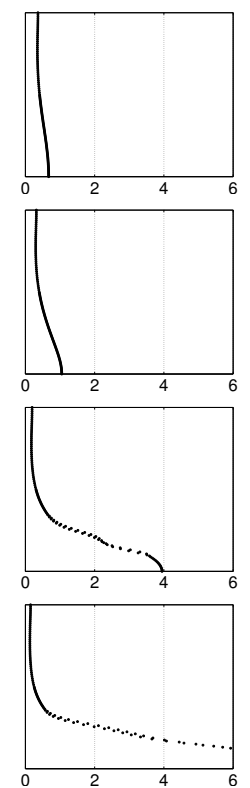

FIG. 7. Problem II: Snapshots of axially symmetric simulation of a sphere pushed through shear thickening fluid at times $t=2.21$ (a), $t=4.40$ (b), $t=4.90$ (c), $t=5.62$ (d) and $t=5.86$ (e). Shown in the left column are the rigid sphere, the thickened volume and the flow field (arrows). The right column shows the normal traction acting on the right domain boundary. As can be seen, force from the motion of the sphere is transmitted through the thickened volume to the right boundary.

in Figure 8. Note that when the system is close to steady state, only a small number of Newton iterations is required per time step.

Figure 9 shows snapshots from the time evolution of the flow field. The solidification front grows when forcing is applied, i.e., for $t \in[0,0.6 T]$, and disappears afterwards. The growing blob of thickened fluid and the material that moves down with it transmits the applied boundary force deeply into the fluid volume. This is a possible explanation why high particle-to-fluid volume ratio dispersion can withhold strong boundary forces for short times, which, for instance, allows running over pools filled with mixtures of water and cornstarch [37, 40].

\section{Conclusions and perspectives}

We proposed a nonsmooth continuum mechanical Stokes flow model that incorporates so-called discontinuous shear thickening at high strain rates, a behavior that can be observed in dispersions with high particle-to-fluid volume fractions. Due to the nonsmooth constitutive relation, the flow changes its viscosity at a free boundary. Despite the nonsmooth strain-rate-to-stress relation, 


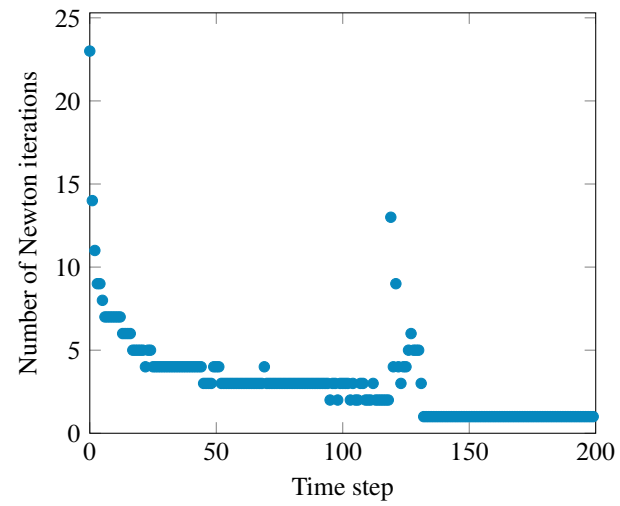

FIG. 8. Problem III: Number of Newton iterations in each time step for $g=1$ and $v=10^{3}$. The increase in the number of iterations at the 120th time step, which corresponds to time $t=0.03$ is due to the surface forcing, which is turned off at that time (see 41)
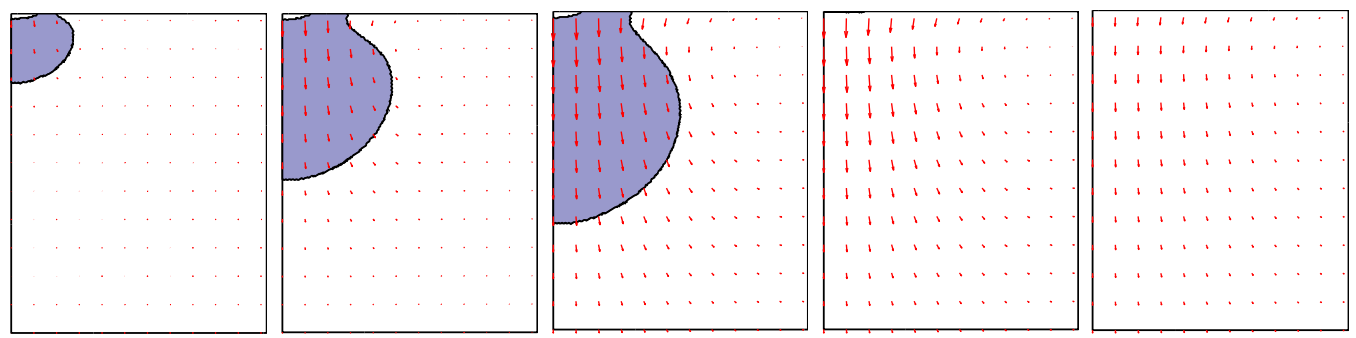

FIG. 9. Problem III: Time evolution of solidified volume and the flow field (arrows) at time steps 1, 21, 111, 121 and 151 out of 200 steps (from left to right). Shown is the region $[0,0.08] \times[0,0.1] \subset \Omega$.

additional solution regularity is proved and, after proper discretization, the problem can be solved efficiently by a locally fast converging second-order method. Numerical simulations show that the flow and the free boundary behave qualitatively similar to what has recently been observed in experiments with fluid-particle suspensions such as cornstarch-water mixtures.

Several questions and possibilities for future research arise from this work. An interesting theoretical question is whether it is possible to show regularity of the free boundary, which occurs in the proposed model. Moreover, finding optimal discretizations for $(\mathrm{P})$ is a possibility for future research. Since we use piecewise constant finite elements for the discretization of the components of the dual tensor $\boldsymbol{\tau}$, we cannot expect optimal convergence of the numerical discretization. Three-dimensional simulations, which require iterative solvers and preconditioners for the varying viscosity Stokes systems that arise in each semismooth Newton step are another natural extension of this work. Finally, the interaction of solids with a discontinuously shear thickening fluid such as the settling of a sphere [38] could be an interesting problem. A possibility to realize such fully coupled fluid-solid problems are fictitious domain methods, which have proven successful in simulations of viscoplastic fluids interacting with solid structures [19]. 
Acknowledgments. The authors would like to thank Michael Shelley and Noemi Petra for helpful discussions and comments. This work was partially supported by a J. Tinsley Oden fellowship, by the Alexander von Humboldt Foundation, by the Escuela Politécnica Nacional de Quito and by the U.S. National Science Foundation (NSF) Cyber-enabled Discovery and Innovation (CDI) program under awards CMS-1028889 and OPP-0941678.

\section{Appendix}

\section{A. Proofs}

\section{Proof of Lemma 4.3}

The first and second derivatives of $m_{\delta}$ are given by

$$
\begin{aligned}
\frac{\partial m_{\delta}(\boldsymbol{\varepsilon})}{\partial \boldsymbol{\varepsilon}}[\boldsymbol{\varphi}] & =\max _{\delta}(0,|\boldsymbol{\varepsilon}|-g) \mathbf{1}_{\delta}(|\boldsymbol{\varepsilon}|-g) \frac{\boldsymbol{\varepsilon}}{|\boldsymbol{\varepsilon}|}: \boldsymbol{\varphi}, \\
\frac{\partial^{2} m_{\delta}(\boldsymbol{\varepsilon})}{\partial \boldsymbol{\varepsilon}^{2}}[\zeta, \boldsymbol{\varphi}] & =\left(\mathbf{1}_{\delta}^{2}(|\boldsymbol{\varepsilon}|-g) \frac{\boldsymbol{\varepsilon}}{|\boldsymbol{\varepsilon}|}: \zeta\right) \frac{\boldsymbol{\varepsilon}}{|\boldsymbol{\varepsilon}|}: \boldsymbol{\varphi}+\max _{\delta}(0,|\boldsymbol{\varepsilon}|-g) \frac{\mathbf{1}_{\delta}(|\boldsymbol{\varepsilon}|-g)}{|\boldsymbol{\varepsilon}|} \zeta: \boldsymbol{\varphi} \\
& +\max _{\delta}(0,|\boldsymbol{\varepsilon}|-g) \chi_{\delta_{\delta}}\left(\frac{-3}{4 \delta^{3}}(|\boldsymbol{\varepsilon}|-g)^{2} \frac{\boldsymbol{\varepsilon}}{|\boldsymbol{\varepsilon}|}: \zeta+\frac{3}{4 \delta} \frac{\boldsymbol{\varepsilon}}{|\boldsymbol{\varepsilon}|^{2}}: \zeta\right) \boldsymbol{\varepsilon}: \boldsymbol{\varphi} \\
& -\max _{\delta}(0,|\boldsymbol{\varepsilon}|-g)\left(\mathbf{1}_{\delta}(|\boldsymbol{\varepsilon}|-g) \frac{\boldsymbol{\varepsilon}}{|\boldsymbol{\varepsilon}|^{3}}: \zeta\right) \boldsymbol{\varepsilon}: \boldsymbol{\varphi},
\end{aligned}
$$

where $\chi_{\delta_{\delta}}=1$ if ||$\varepsilon|-g| \leqslant \delta$ and $\chi_{\delta_{\delta}}=0$ otherwise. From the explicit form of the second derivative, (25) follows.

\section{Proof of Lemma 4.4}

Note that the derivative of $\max _{\delta}$ is given by

$$
\mathbf{1}_{\delta}(x)= \begin{cases}1 & \text { if } x \geqslant \delta, \\ -\frac{1}{4 \delta^{3}} x^{3}+\frac{3}{4 \delta} x+\frac{1}{2} & \text { if }|x| \leqslant \delta, \\ 0 & \text { if } x \leqslant-\delta .\end{cases}
$$

For $|x|>\delta, \max _{\delta}(0, x)-\max (0, x) \mathbf{1}_{\delta}(x)=0$. For $0 \leqslant x<\delta$, one obtains the estimate

$$
\begin{aligned}
\left|\max _{\delta}(0, x)-\max (0, x) \mathbf{1}_{\delta}(x)\right| & =\left|x-\left(\frac{x^{4}}{16 \delta^{3}}-\frac{3 x^{2}}{8 \delta}-\frac{x}{2}-\frac{3 \delta}{16}\right)\left(\frac{x^{3}}{4 \delta^{3}}-\frac{3 x}{4 \delta}-\frac{1}{2}\right)\right| \\
& \leqslant \delta+\frac{3}{2}\left(\frac{\delta}{16}+\frac{3 \delta}{8}+\frac{\delta}{2}+\frac{3 \delta}{16}\right) \stackrel{\delta \rightarrow 0}{\longrightarrow} 0 .
\end{aligned}
$$

Finally, for $-\delta<x<0$, one finds

$$
\begin{aligned}
\left|\max _{\delta}(0, x)-\max (0, x) \mathbf{1}_{\delta}(x)\right| & \leqslant\left|-\frac{x^{4}}{16 \delta^{3}}+\frac{3 x^{2}}{8 \delta}+\frac{x}{2}+\frac{3 \delta}{16}\right|\left|-\frac{x^{3}}{4 \delta^{3}}+\frac{3 x}{4 \delta}+\frac{1}{2}\right| \\
& \leqslant \frac{27}{16} \delta \stackrel{\delta \rightarrow 0}{\longrightarrow} 0
\end{aligned}
$$

which proves the claim for all $x \in \mathbb{R}$. 
Proof of Lemma 4.5

We argue by contradiction, i.e., we suppose that the claim is false. Then, for any $c_{0}>0$ and $t \in(0,1)$ we can find a sequence $\left\{\delta_{k}\right\}$, with $\delta_{k} \rightarrow 0$, and corresponding minimizers of $\oint_{\delta}$, denoted by $\left\{\boldsymbol{v}_{k}\right\}$, and balls $\left\{B_{r_{k}}\left(x_{k}\right)\right\} \subset \Omega$ such that

$$
\begin{aligned}
& \left|\left(\varepsilon \boldsymbol{v}_{k}\right)_{x_{k}, r_{k}}\right| \leqslant \sigma, \\
& \epsilon_{k}^{2}:=V\left(v_{k}, B_{r_{k}}\left(x_{k}\right)\right) \searrow 0 \text { as } k \rightarrow \infty, \\
& \mathcal{V}\left(\boldsymbol{v}_{k}, B_{t r_{k}}\left(x_{k}\right)\right)>c_{0} t^{2} \epsilon_{k}^{2} .
\end{aligned}
$$

Defining $\vartheta_{k}:=\left(\varepsilon \boldsymbol{v}_{k}\right)_{x_{k}, r_{k}}$ and proceeding as in [14, Lem. 2.2], we may define rescaled functions $\left\{\boldsymbol{u}_{k}\right\}$, for which we obtain

$$
\begin{aligned}
& f_{B_{1}}\left|\boldsymbol{\varepsilon} \boldsymbol{u}_{k}\right|^{2} d x \leqslant 1, \\
& \boldsymbol{u}_{k} \rightarrow \boldsymbol{v} \text { in } H^{1}\left(B_{1}\right)^{N}, \\
& \boldsymbol{\vartheta}_{k} \rightarrow \boldsymbol{\vartheta}_{0},
\end{aligned}
$$

where $B_{1}:=B_{1}\left(x_{0}\right), v \in H^{1}\left(B_{1}\right)^{N}$ and $\vartheta_{0} \in \mathbb{R}^{n \times n}$. From (25), we have that there exists a constant $C>0$, which is independent of $\delta$ such that

$$
\left|\frac{\partial^{2} m_{\delta}\left(\varepsilon \boldsymbol{u}_{\delta}\right)}{\partial \boldsymbol{\varepsilon}^{2}}\right| \leqslant C
$$

Therefore, proceeding as in [14, pp. 1008-1009], the convergence result for $\varepsilon \boldsymbol{u}_{k}$ is improved and we obtain that

$$
\varepsilon \boldsymbol{u}_{k} \rightarrow \varepsilon \boldsymbol{v} \text { strongly in } L_{l o c}^{2}\left(B_{1}\right)^{N} \text {. }
$$

To obtain a contradiction, we will make use of Campanato-type estimates, which follow directly once a blow-up equation is verified at the limit (see [14, Lem. 1.5]). In our case this equation takes the following form:

$$
\begin{aligned}
2 f_{B_{1}} \varepsilon \boldsymbol{v}: \varepsilon \varphi d x=-f_{B_{1}} \chi_{Q}( & \left.\frac{\boldsymbol{\vartheta}_{0}}{\left|\boldsymbol{\vartheta}_{0}\right|}: \varepsilon \boldsymbol{v}\right) \frac{\boldsymbol{\vartheta}_{0}}{\left|\boldsymbol{\vartheta}_{0}\right|}: \varepsilon \boldsymbol{\varphi} \\
& \quad+\max \left(0,\left|\boldsymbol{\vartheta}_{0}\right|-g\right)\left[\frac{\varepsilon \boldsymbol{v}}{\left|\boldsymbol{\vartheta}_{0}\right|}-\left(\frac{\boldsymbol{\vartheta}_{0}}{\left|\boldsymbol{\vartheta}_{0}\right|^{3}}: \varepsilon \boldsymbol{v}\right) \boldsymbol{\vartheta}_{0}\right]: \varepsilon \boldsymbol{\varphi} d x
\end{aligned}
$$

where $\varphi \in V$ and $Q:=\left\{x \in \Omega:\left|\vartheta_{0}\right| \geqslant g\right\}$.

To prove (47), we proceed along the lines of [14, Lem. 2.5]. The main difference is due to the form of the regularized functional. In this respect, the proof is finished once we verify the convergence of

$$
\mathrm{II}_{k}:=\epsilon_{k}^{-1} \int_{B_{1}}\left[\frac{\partial m_{\delta}\left(\epsilon_{k} \boldsymbol{\varepsilon} \boldsymbol{u}_{k}+\vartheta_{k}\right)}{\partial \boldsymbol{\varepsilon}}-\frac{\partial m_{\delta}\left(\boldsymbol{\vartheta}_{k}\right)}{\partial \boldsymbol{\varepsilon}}\right]: \varepsilon \boldsymbol{\varphi} d x,
$$


as $k \rightarrow \infty$. The integral $\mathrm{II}_{k}$ may be decomposed as $\mathrm{II}_{k}=\mathrm{II}_{k}^{1}+\mathrm{II}_{k}^{2}$, where

$$
\begin{aligned}
\mathrm{II}_{k}^{1} & :=\int_{B_{1}} \int_{0}^{1}\left[\frac{\partial^{2} m_{\delta}\left(\boldsymbol{\vartheta}_{k}+s \epsilon_{k} \varepsilon \boldsymbol{u}_{k}\right)}{\partial \boldsymbol{\varepsilon}^{2}}-\frac{\partial^{2} m_{\delta}\left(\boldsymbol{\vartheta}_{k}\right)}{\partial \boldsymbol{\varepsilon}^{2}}\right] \varepsilon \boldsymbol{u}_{k}: \varepsilon \boldsymbol{\varphi} d s d x \\
\mathrm{II}_{k}^{2} & :=\int_{B_{1}} \frac{\partial^{2} m_{\delta}\left(\boldsymbol{\vartheta}_{k}\right)}{\partial \boldsymbol{\varepsilon}^{2}} \boldsymbol{\varepsilon} \boldsymbol{u}_{k}: \varepsilon \boldsymbol{\varphi} d x .
\end{aligned}
$$

Thanks to (44c), (45), the pointwise convergence of $\partial^{2} m_{\delta}(\boldsymbol{\varepsilon}) / \partial \boldsymbol{\varepsilon}^{2}$ for $\delta \rightarrow 0$, and Lebesgue's dominated convergence theorem, it follows that

$$
\mathrm{II}_{k}^{2} \rightarrow \int_{B_{1}} \chi_{Q}\left(\frac{\boldsymbol{\vartheta}_{0}}{\left|\boldsymbol{\vartheta}_{0}\right|}: \varepsilon \boldsymbol{v}\right) \frac{\boldsymbol{\vartheta}_{0}}{\left|\boldsymbol{\vartheta}_{0}\right|}: \mathcal{\varepsilon} \boldsymbol{\varphi}+\max \left(0,\left|\boldsymbol{\vartheta}_{0}\right|-g\right)\left[\frac{\varepsilon \boldsymbol{v}}{\left|\boldsymbol{\vartheta}_{0}\right|}-\left(\frac{\boldsymbol{\vartheta}_{0}}{\left|\boldsymbol{\vartheta}_{0}\right|^{3}}: \varepsilon \boldsymbol{v}\right) \boldsymbol{\vartheta}_{0}\right]: \varepsilon \boldsymbol{\varphi} d x,
$$

where $Q:=\left\{x \in \Omega:\left|\vartheta_{0}\right| \geqslant g\right\}$. To finish the proof it remains to show that $\mathrm{II}_{k}^{1} \rightarrow 0$ as $k \rightarrow \infty$. Since $\epsilon_{k} \varepsilon \boldsymbol{u}_{k} \rightarrow 0$ in $L^{2}\left(B_{1}\right)^{N}$ with pointwise almost everywhere convergence in $B_{1}$ (see [14, eq. (2.24)]) we may apply Egorov's theorem to $\mathrm{II}_{k}^{\alpha}$ (see [14, p. 1002]) to obtain this result.

From Campanato estimates [1, Lem. 5.1], there exists a constant $c^{*}$ such that

$$
f_{B_{t}}\left|\varepsilon v-(\varepsilon v)_{t}\right|^{2} d x \leqslant c^{*} t^{2} f_{B_{1}}\left|\varepsilon v-(\varepsilon v)_{1}\right|^{2} d x
$$

where $c^{*}$ depends only on the ellipticity constant for the blow-up equation (47) and $(\varepsilon v)_{1}:=$ $\frac{1}{B_{1}} \int_{B_{1}} \varepsilon v d x$. In particular, we may choose $c_{0}:=2 c^{*}$.

From (43) and the definition of $\boldsymbol{u}_{k}$ (see [14, Lem. 2.2]) it follows that $v\left(\boldsymbol{u}_{k}, B_{t}\right)>c_{0} t^{2}$. The strong convergence $\varepsilon \boldsymbol{u}_{k} \rightarrow \boldsymbol{\varepsilon} \boldsymbol{v}$ (see (46)) implies that

$$
f_{B_{t}}\left|\varepsilon v-(\varepsilon v)_{t}\right|^{2} d x \geqslant c_{0} t^{2}
$$

From (44a) and (44b), we get that

$$
f_{B_{1}}|\mathcal{E} \boldsymbol{v}|^{2} d x \leqslant \liminf _{k \rightarrow \infty} f_{B_{1}}\left|\mathcal{E} \boldsymbol{u}_{k}\right|^{2} d x \leqslant 1,
$$

which, combined with (50), implies that

$$
f_{B_{t}}\left|\varepsilon v-(\varepsilon v)_{t}\right|^{2} d x \geqslant c_{0} t^{2} f_{B_{1}}\left|\mathcal{E v}-(\varepsilon v)_{1}\right|^{2} d x
$$

Comparing the last result with (49), the desired contradiction is obtained.

\section{REFERENCES}

1. Bildhauer, M., \& Fuchs, M., Variants of the Stokes problem: the case of anisotropic potentials. Journal of Mathematical Fluid Mechanics 5 (2003), 364-402. Zbl1072. 76019 MR2004292

2. Boyer, F., Guazzelli, É., \& Pouliquen, O., Unifying suspension and granular rheology. Physical Review Letters 10718 (2011), 188301. 
3. BREZIS, H., Multiplicateur de Lagrange en torsion elasto-plastique. Archive for rational mechanics and analysis 49 (1972), 32-40. Zbl0265. 35021 MR0346346

4. Brown, E., Forman, N., Orellana, C., Zhang, H., Maynor, B., Betts, D., DeSimone, J., \& JAEGER, H., Generality of shear thickening in dense suspensions. Nature Materials 9 (2010), 220-224.

5. Brown, E., \& JAEGER, H. M., The role of dilation and confining stresses in shear thickening of dense suspensions. Journal of Rheology 56 (2012), 875-923.

6. Burstedde, C., Ghattas, O., Stadler, G., Tu, T., \& Wilcox, L. C., Parallel scalable adjointbased adaptive solution for variable-viscosity Stokes flows. Computer Methods in Applied Mechanics and Engineering 198 (2009), 1691-1700. Zbl1227.76027

7. De los Reyes, J. C., \& GonzÁlez, S., Numerical simulation of two-dimensional Bingham fluid flow by semismooth Newton methods. Journal of Computational and Applied Mathematics 235 (2010), 11-32. Zbl05804863

8. Elliott, C. M., \& OCKENDON, J. R., Weak and variational methods for moving boundary problems. Pitman Pub. (1982). Zbl0476. 35080 MR0650455

9. Elman, H. C., Silvester, D. J., \& Wathen, A. J., Finite Elements and Fast Iterative Solvers with applications in incompressible fluid dynamics. Oxford University Press, Oxford (2005). Zb11083.76001 MR2155549

10. Evans, L. C., Partial differential equations, second ed., vol. 19 of Graduate Studies in Mathematics. American Mathematical Society, Providence, RI, 2010. Zbl1194.35001 MR2597943

11. Fall, A., Bertrand, F., Ovarlez, G., \& Bonn, D., Shear thickening of cornstarch suspensions. Journal of Rheology 56 (2012), 575.

12. Fall, A., Huang, N., Bertrand, F., Ovarlez, G., \& Bonn, D., Shear thickening of cornstarch suspensions as a reentrant jamming transition. Physical Review Letters 100 (Jan 2008), 018301.

13. Frehse, J., Málek, J., \& Steinhauer, M., An existence result for fluids with shear dependent viscosity-steady flows. Nonlinear Analysis: Theory, Methods \& Applications 30 (1997), 3041-3049. Zbl0902.35089 MR1602949

14. Fuchs, M., Grotowski, J. F., \& Reuling, J., On variational models for quasi-static Bingham fluids. Mathematical Methods in the Applied Sciences 19 (1996), 991-1015. Zb10857.76006 MR1402153

15. Fuchs, M., \& SEREgIN, G., Regularity results for the quasi-static Bingham variational inequality in dimensions two and three. Mathematische Zeitschrift 227 (1998), 525-541. Zb10899. 76044 MR1612689

16. FUCHS, M., \& SEREGIN, G., Variational methods for problems from plasticity theory and for generalized Newtonian fluids, vol. 1749 of Lecture Notes in Mathematics. Springer-Verlag, Berlin (2000). Zb10964. 76003 MR1810507

17. Glowinski, R., Lions, J. L., \& Trémolières, R., Numerical Analysis of Variational Inequalities. North-Holland, Amsterdam (1981). Zb10463.65046 MR0635927

18. Glowinski, R., Pan, T.-W., Hesla, T., Joseph, D., \& Periaux, J., A distributed Lagrange multiplier/fictitious domain method for flows around moving rigid bodies: application to particulate flow. International Journal for Numerical Methods in Fluids 30 (1999), 1043-66. Zb10971. 76046

19. Glowinski, R., \& XU, J. Numerical Methods for Non-Newtonian Fluids: Special Volume, vol. 16 of Handbook of numerical analysis. North-Holland, 2011. Zbl1216.76001

20. Grinevich, P., \& Olshanskit, M., An iterative method for the Stokes-type problem with variable viscosity. SIAM Journal on Scientific Computing 31 (2009), 3959-3978. Zb105801906 MR2563521

21. Hintermüller, M., Ito, K., \& KUnisch, K., The primal-dual active set strategy as a semi-smooth Newton method. SIAM Journal on Optimization 13 (2003), 865-888. Zbl1080 . 90074 MR1972219

22. Hoffman, R. L., Explanations for the cause of shear thickening in concentrated colloidal suspensions. Journal of Rheology 42 (1998), 111-123.

23. Idone, G., Maugeri, A., \& Vitanza, C., Variational inequalities and the elastic-plastic torsion problem. Journal of optimization theory and applications 117 (2003), 489-501. Zbl1038.49009 MR1989924 
24. Kaplickỳ, P., Málek, J., \& StARÁ, J., $\mathrm{C}^{1, \alpha}$-solutions to a class of nonlinear fluids in the 2D stationary Dirichlet problem. Journal of Mathematical Sciences 109 (2002), 1867-1893. Zb10978. 35046 MR1754359

25. KIKUCHI, N., \& ODEN, J. T., Contact problems in elasticity: a study of variational inequalities and finite element methods. No. 8. SIAM (1988). Zb10685.73002 MR0961258

26. Ladyzhenskaya, O. A., The mathematical theory of viscous incompressible flow. Second English edition. Mathematics and its Applications, Vol. 2. Gordon and Breach Science Publishers, New York (1969). Zb10184.52603 MR0254401

27. Lions, J., Quelques méthodes de résolution des problemes aux limites non linéaires, vol. 76. Dunod Paris (1969). Zb10189.40603 MR0259693

28. LiU, B., Shelley, M., \& ZHANG, J., Focused force transmission through an aqueous suspension of granules. Physical Review Letters 10518 (2010), 188301.

29. Majumdar, S., Krishnaswamy, R., \& Sood, A., Discontinuous shear thickening in confined dilute carbon nanotube suspensions. Proceedings of the National Academy of Sciences 10822 (2011), 8996.

30. May, D. A., \& Moresi, L., Preconditioned iterative methods for Stokes flow problems arising in computational geodynamics. Physics of the Earth and Planetary Interiors 171 (2008), 33-47.

31. Mifflin, R., Semismooth and semiconvex functions in constrained optimization. SIAM Journal on Control and Optimization 15 (1977), 959-972. Zb10376.90081 MR0461556

32. QI, L., \& SUN, J., A nonsmooth version of Newton's method. Mathematical Programming 58 Ser. A (1993), 353-367. Zb10780.90090 MR1216791

33. Rajagopal, K. R., Mechanics of non-Newtonian fluids. Pitman Research Notes in Mathematics Series (1993), 129-162. Zbl0818.76003 MR1268237

34. Rodrigues, J. F., On the mathematical analysis of thick fluids. Tech. Rep. NI14031-FRB, Isaac Newton Institute for Mathematical Sciences, 2014.

35. Sazhenkov, S., The problem of motion of rigid bodies in a non-Newtonian incompressible fluid. Siberian Matematical Journal 39 (1998), 146-160. Zbl0938. 35120 MR1623751

36. Slawig, T., Distributed control for a class of non-Newtonian fluids. Journal of Differential Equations 2191 (2005), 116-143. Zbl1086. 49001 MR2181032

37. VAn Hecke, M., Soft matter: Running on cornflour. Nature 487 (2012), 174-175.

38. von Kann, S., SnoeiJer, J. H., Lohse, D., \& VAn der Meer, D., Nonmonotonic settling of a sphere in a cornstarch suspension. Physical Review E 84 (Dec 2011), 060401.

39. Wagner, N., \& Brady, J., Shear thickening in colloidal dispersions. Physics Today 62 (2009), 27-32.

40. WAitukaitis, S., \& JAEGER, H., Impact-activated solidification of dense suspensions via dynamic jamming fronts. Nature 4877406 (2012), 205-209. 